\title{
THE SLOPE OF THE BLACK HOLE MASS VERSUS VELOCITY DISPERSION CORRELATION
}

\author{
Scott Tremaine, ${ }^{1}$ Karl Gebhardt ${ }^{2}$ Ralf Bender,${ }^{3}$ Gary Bower, ${ }^{4}$ Alan Dressler,${ }^{5}$ S. M. Faber,${ }^{6}$ \\ Alexei V. Filippenko, ${ }^{7}$ Richard Green, ${ }^{8}$ Carl Grillmair, ${ }^{9}$ Luis C. Ho, ${ }^{5}$ John Kormendy, ${ }^{2}$ \\ Tod R. Lauer, ${ }^{8}$ John Magorrian, ${ }^{10}$ Jason Pinkney, ${ }^{11}$ And Douglas Richstone ${ }^{11}$ \\ Received 2002 January 17; accepted 2002 April 3
}

\begin{abstract}
Observations of nearby galaxies reveal a strong correlation between the mass of the central dark object $M_{\mathrm{BH}}$ and the velocity dispersion $\sigma$ of the host galaxy, of the form $\log \left(M_{\mathrm{BH}} / M_{\odot}\right)=\alpha+\beta \log \left(\sigma / \sigma_{0}\right)$; however, published estimates of the slope $\beta$ span a wide range (3.75-5.3). Merritt \& Ferrarese have argued that low slopes $(\lesssim 4)$ arise because of neglect of random measurement errors in the dispersions and an incorrect choice for the dispersion of the Milky Way Galaxy. We show that these explanations and several others account for at most a small part of the slope range. Instead, the range of slopes arises mostly because of systematic differences in the velocity dispersions used by different groups for the same galaxies. The origin of these differences remains unclear, but we suggest that one significant component of the difference results from Ferrarese \& Merritt's extrapolation of central velocity dispersions to $r_{e} / 8$ ( $r_{e}$ is the effective radius) using an empirical formula. Another component may arise from dispersion-dependent systematic errors in the measurements. A new determination of the slope using 31 galaxies yields $\beta=4.02 \pm 0.32, \alpha=8.13 \pm 0.06$ for $\sigma_{0}=200 \mathrm{~km} \mathrm{~s}^{-1}$. The $M_{\mathrm{BH}}-\sigma$ relation has an intrinsic dispersion in $\log M_{\mathrm{BH}}$ that is no larger than $0.25-0.3$ dex and may be smaller if observational errors have been underestimated. In an appendix, we present a simple kinematic model for the velocity-dispersion profile of the Galactic bulge.
\end{abstract}

Subject headings: black hole physics — galaxies: bulges — galaxies: fundamental parameters — galaxies: nuclei — Galaxy: bulge — Galaxy: kinematics and dynamics

\section{INTRODUCTION}

Observations of the centers of nearby early-type galaxies (ellipticals, lenticulars, and spiral bulges) show that most or all contain massive dark objects (hereafter "black holes"). The masses of these objects are consistent with the density of quasar remnants expected from energy arguments (Sołtan 1982; Fabian \& Iwasawa 1999; Yu \& Tremaine 2002). There appears to be a strong correlation between the mass $M_{\mathrm{BH}}$ of the black hole and the velocity dispersion $\sigma$ of the host galaxy, of the form ${ }^{12}$

$$
\log \left(M_{\mathrm{BH}} / M_{\odot}\right)=\alpha+\beta \log \left(\sigma / \sigma_{0}\right),
$$

where $\sigma_{0}$ is some reference value (here chosen to be

\footnotetext{
${ }^{1}$ Princeton University Observatory, Peyton Hall, Princeton, NJ 08544; tremaine@astro.princeton.edu.

2 Department of Astronomy, University of Texas, RLM 15.308, Austin, TX78712; gebhardt@astro.as.utexas.edu,kormendy@astro.as.utexas.edu.

${ }^{3}$ Universitäts-Sternwarte, Scheinerstrasse 1, Munich 81679, Germany; bender@usm.uni-muenchen.de.

${ }^{4}$ Computer Sciences Corporation, Space Telescope Science Institute, 3700 San Martin Drive, Baltimore, MD 21218; bower@stsci.edu.

5 Observatories of the Carnegie Institution of Washington, 813 Santa Barbara Street, Pasadena, CA 91101; dressler@ociw.edu, lho@ociw.edu.

${ }^{6}$ UCO/Lick Observatories, University of California, Santa Cruz, CA 95064; faber@ucolick.org.

${ }^{7}$ Department of Astronomy, University of California, Berkeley, CA94720-3411; alex@astro.berkeley.edu.

${ }^{8}$ National Optical Astronomy Observatory, P.O. Box 26732, Tucson, AZ 85726; green@noao.edu, lauer@noao.edu.

${ }^{9}$ SIRTF Science Center, MS 220-6, 1200 East California Boulevard, Pasadena, CA 91125; carl@ipac.caltech.edu.

${ }^{10}$ Department of Physics, University of Durham, Rochester Building, Science Laboratories, South Road, Durham DH1 3LE, UK; john.magorrian@durham.ac.uk.

${ }^{11}$ Department of Astronomy, Dennison Building, University of Michigan, Ann Arbor, MI 48109; jpinkney@astro.lsa.umich.edu, dor@astro.lsa.umich.edu.

12 All logarithms in this paper are base 10 .
}

$\left.\sigma_{0}=200 \mathrm{~km} \mathrm{~s}^{-1}\right)$. The first published estimates of the slope $\beta, 5.27 \pm 0.40$ (L. Ferrarese \& D. Merritt 2000, astro-ph/ $0006053 \mathrm{v} 1$ ) and $3.75 \pm 0.3$ (Gebhardt et al. 2000a), differed by 3 standard deviations. Subsequently, Ferrarese \& Merritt (hereafter FM) revised their slope downward, to $4.8 \pm 0.5$ (Ferrarese \& Merritt 2000), $4.72 \pm 0.36$ (Merritt \& Ferrarese 2001a), $4.65 \pm 0.48$ (Merritt \& Ferrarese 2001b), and then $4.58 \pm 0.52$ (Ferrarese 2002). Although the discrepancy between the estimate by Gebhardt et al. (hereafter the Nukers) and the estimates by FM has declined monotonically with time and is now only 1.4 standard deviations, it is still worthwhile to understand the reasons behind it. In particular, the slope is the most important point of comparison to theoretical models that attempt to explain the $M_{\mathrm{BH}^{-}} \sigma$ relation (Adams, Graff, \& Richstone 2001; Burkert \& Silk 2001; Haehnelt \& Kauffmann 2000; Ostriker 2000).

This paper has three main goals. (1) In $\S \S 2-4$ we explore the reasons for the wide range in estimated slopes of the $M_{\mathrm{BH}}-\sigma$ relation. In $\S 2$ we focus on the statistical techniques used to estimate slopes by the two groups; we argue that the estimator used by the Nukers is more accurate but that the choice of estimator cannot explain most of the differences in slope between FM and the Nukers. In $\S 3$ we describe the data sets used by the two groups. In $\S 4$ we examine several explanations that have been proposed for the slope range, including the neglect of random measurement errors in the dispersions, the dispersion used for the Milky Way, and differences in sample selection, and show that none of these is viable. We argue instead that the slope range reflects systematic differences in the velocity dispersion measurements used by the two groups. (2) In $\S 5$ we present a new analysis of the $M_{\mathrm{BH}}-\sigma$ relation using recent data. (3) Finally, in the Appendix we model the velocity-dispersion profile of the Milky Way bulge, which helps to fix the low-mass end of the $M_{\mathrm{BH}}-\sigma$ relation. 


\section{THE FITTING ALGORITHM}

The data consist of $N$ galaxies with measured black hole masses, velocity dispersions, and associated uncertainties. We assume that there is an underlying relation of the form

$$
y=\alpha+\beta x,
$$

where $y=\log \left(M_{\mathrm{BH}} / M_{\odot}\right), x=\log \left(\sigma / \sigma_{0}\right)$. We assume that the measurement errors are symmetric in $x$ and $y$ with rms values $\epsilon_{x i}$ and $\epsilon_{y i}$ for galaxy $i$. The goal is to estimate the best-fit values of $\alpha$ and $\beta$ and their associated uncertainties.

The Nukers and FM use two quite different estimators. In this section we review the assumptions inherent in the two estimators and their respective advantages and disadvantages. In subsequent sections we usually give results for both estimators; we find that the differences are significant but not large enough to explain the slope range.

The Nukers' estimate is based on minimizing

$$
\chi^{2} \equiv \sum_{i=1}^{N} \frac{\left(y_{i}-\alpha-\beta x_{i}\right)^{2}}{\epsilon_{y i}^{2}+\beta^{2} \epsilon_{x i}^{2}}
$$

(e.g., Press et al. 1992, whose procedures we use). The " $1 \sigma$ " uncertainties in $\alpha$ and $\beta$ are given by the maximum range of $\alpha$ and $\beta$ for which $\chi^{2}-\chi_{\min }^{2} \leq 1$. An attractive feature of this approach is that the variables $x$ and $y$ are treated symmetrically; in other words, if we set $\tilde{\beta}=1 / \beta, \tilde{\alpha}=-\alpha / \beta$, equation (3) can be rewritten in the form

$$
\chi^{2} \equiv \sum_{i=1}^{N} \frac{\left(x_{i}-\tilde{\alpha}-\tilde{\beta} y_{i}\right)^{2}}{\epsilon_{x i}^{2}+\tilde{\beta}^{2} \epsilon_{y i}^{2}}
$$

which has the same form as equation (3) if $x \leftrightarrow y, \alpha \leftrightarrow \tilde{\alpha}$, and $\beta \leftrightarrow \tilde{\beta}$. This symmetry ensures that we are not assuming (for example) that $y$ is the dependent variable and $x$ is the independent variable in the correlation; this agnosticism is important because we do not understand the physical mechanism that links black hole mass to dispersion. We call estimators of this kind " $\chi^{2}$ estimators" and denote them by $\alpha_{\chi}, \beta_{\chi}$.

One limitation is that this approach does not account for any intrinsic dispersion in the $M_{\mathrm{BH}}-\sigma$ relation (i.e., dispersion due to the galaxies themselves rather than to measurement errors). Thus, for example, one or two very precise measurements with small values of $\epsilon_{x i}$ and $\epsilon_{y i}$ can dominate $\chi^{2}$, even though the large weight given to these observations is unrealistic if the intrinsic dispersion is larger than the measurement errors. There are two heuristic approaches that address this concern. (1) Simply set $\epsilon_{y i} \equiv \epsilon_{y}=$ constant, corresponding to the same fractional uncertainty in all the black hole mass estimates. The value of $\epsilon_{y}$ is adjusted so that the value of $\chi^{2}$ per degree of freedom is equal to its expectation value of unity. This approach was adopted by Gebhardt et al. (2000a). (2) Replace $\epsilon_{y i}$ by $\left(\epsilon_{y i}^{2}+\epsilon_{0}^{2}\right)^{1 / 2}$, where the unknown constant $\epsilon_{0}$, which represents the intrinsic dispersion, is adjusted so that the value of $\chi^{2}$ per degree of freedom is unity. The second procedure is preferable if and only if the individual error estimates $\epsilon_{y i}$ are reliable. We use both approaches in $\S 5.2$.

FM use the estimator

$$
\beta_{\mathrm{AB}}=\frac{\sum_{i=1}^{N}\left(y_{i}-\langle y\rangle\right)\left(x_{i}-\langle x\rangle\right)}{\sum_{i=1}^{N}\left(x_{i}-\langle x\rangle\right)^{2}-\sum_{i=1}^{N} \epsilon_{x i}^{2}}, \quad \alpha_{\mathrm{AB}}=\langle y\rangle-\beta_{\mathrm{AB}}\langle x\rangle ;
$$

here $\langle x\rangle \equiv N^{-1} \sum_{i=1}^{N} x_{i}$ and $\langle y\rangle \equiv N^{-1} \sum_{i=1}^{N} y_{i}$ are the sample means of the two variables. This estimator is described by Akritas \& Bershady (1996), who also provide formulae for the uncertainties in $\alpha$ and $\beta$. The Akritas-Bershady (hereafter $\mathrm{AB}$ ) estimator accounts for measurement uncertainties in both variables and is asymptotically normal and consistent. When $\epsilon_{x i}=0$ and $\epsilon_{y i} \equiv \epsilon_{y}=$ constant, the $\mathrm{AB}$ and $\chi^{2}$ estimators give the same estimates for $\alpha$ and $\beta$ (but not their uncertainties).

Despite its merits, the $\mathrm{AB}$ estimator has several unsettling properties. (1) The measurement errors in velocity dispersion, $\epsilon_{x i}$, enter equation (5) only through the sum $\sum \epsilon_{x i}^{2}$. Thus, for example, a single low-precision measurement can dominate both $\sum \epsilon_{x i}^{2}$ and $\sum_{i=1}^{N}\left(x_{i}-\langle x\rangle\right)^{2}$, rendering the estimator useless, no matter how many high-precision measurements are in the sample. (2) The errors in the black hole mass determinations $\epsilon_{y i}$ do not enter equation (5) at all: all observations are given equal weight, even if some are known to be much less precise than others. (3) We have argued above that the variables $x$ and $y$ should be treated symmetrically, but this is not the case in equation (5). (4) Even if the variables $x_{i}$ are drawn from a Gaussian distribution, there will occasionally be samples for which the denominator of equation (5) is near zero. In this case the estimator $\beta_{\mathrm{AB}}$ will be very large. These occasional large excursions are frequent enough that the variance of $\beta_{\mathrm{AB}}$ in a population of galaxy samples is infinite, no matter how large the number $N$ of data points may be. (5) Figure 1 shows the distribution of estimates of $\beta_{\chi}$ (solid line) and $\beta_{\mathrm{AB}}$ (dashed line) obtained from 100,000 Monte Carlo trials drawn from a population that has $\beta=4.5$ and other parameters similar to the sample FM1, defined below (for details see figure legend). The distribution of $\beta_{\chi}$ is substantially narrower than $\beta_{\mathrm{AB}}$ (note that values of either estimator outside the range of the histogram are plotted in the outermost bins). The estimator $\beta_{\chi}$ has a mean of 4.52 and a standard deviation of 0.36 . The distribution of $\beta_{\mathrm{AB}}$ has a mean of 4.69 , and as stated above, the standard deviation of this mean is infinite. Thus, in this example at least, $\beta_{\mathrm{AB}}$ is both biased and inefficient.

In this paper, we sometimes use a third fitting procedure, which is closely related to principal component analysis (Kendall, Stuart, \& Ord 1983). Suppose that the intrinsic distribution of $x$ and $y$ (the distribution that would be observed in the absence of measurement errors) is a biaxial Gaussian, with major and minor axes having standard deviations $\sigma_{a}$ and $\sigma_{b}$, respectively, and the major axis having slope $\beta \equiv \tan \theta$. If $\sigma_{b}$ were zero, all of the points would lie exactly on a line of slope $\beta$; thus $\sigma_{b}$ characterizes the intrinsic dispersion in the correlation between $x$ and $y$. Let us also assume that the measurement errors are Gaussian, with standard deviations $\epsilon_{x}$ and $\epsilon_{y}$ that are the same for all galaxies. The observed distribution of $x$ and $y$, which is obtained by convolving the intrinsic Gaussian with the measurement errors, is still Gaussian. The shape of this Gaussian is fully described by the three independent components of the symmetric $2 \times 2$ dispersion tensor

$$
\sigma_{i j} \equiv\left\langle\left(x_{i}-\left\langle x_{i}\right\rangle\right)\left(x_{j}-\left\langle x_{j}\right\rangle\right)\right\rangle, \quad i=1,2, \quad j=1,2,
$$

where $\left(x_{1}, x_{2}\right) \equiv(x, y)$ and $\langle\cdot\rangle$ denotes a sample average. In this idealized but plausible model, at most three of the five parameters $\epsilon_{x}, \epsilon_{y}, \sigma_{a}, \sigma_{b}$, and $\beta$ can be determined from the data, no matter how many galaxies we observe. For 


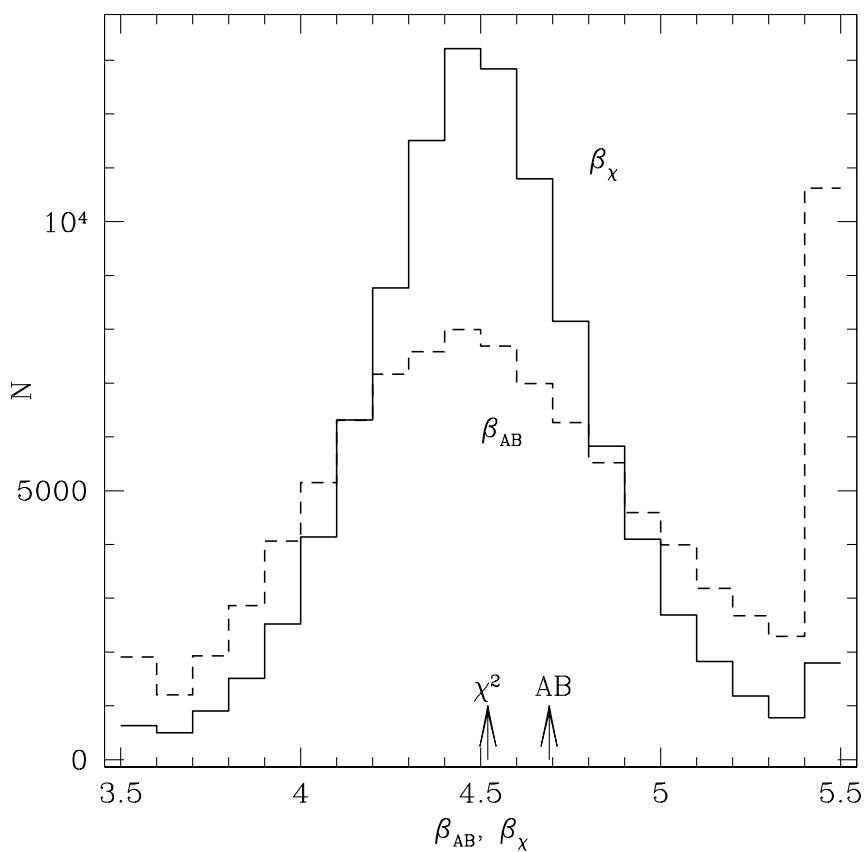

FIG. 1.-Distribution of the estimators $\beta_{\chi}$ (eq. [3]; solid line) and $\beta_{\mathrm{AB}}$ (eq. [5]; dashed line) for 100,000 Monte Carlo simulations of a sample with $\beta=4.5$ that resembles the actual sample FM1 (12 galaxies, distributed as a Gaussian with standard deviation 0.20 in $x$, and Gaussian measurement errors with standard deviations $\epsilon_{x}=0.06, \epsilon_{y}=0.18$ ). Values greater than 5.5 or less than 3.3 are plotted in the outermost bins of the histogram. The sample means are marked by arrows.

example, if $\epsilon_{x}$ and $\epsilon_{y}$ are known, the other parameters can be estimated using the formulae

$$
\begin{gathered}
\tan 2 \theta=\frac{2 \sigma_{x y}}{\sigma_{x x}-\sigma_{y y}+\epsilon_{y}^{2}-\epsilon_{x}^{2}}, \\
\sigma_{b}^{2}=\sigma_{x x}-\epsilon_{x}^{2}-\sigma_{x y} \cot \theta=\sigma_{y y}-\epsilon_{y}^{2}-\sigma_{x y} \tan \theta, \\
\sigma_{a}^{2}=\sigma_{x x}-\epsilon_{x}^{2}+\sigma_{x y} \tan \theta=\sigma_{y y}-\epsilon_{y}^{2}+\sigma_{x y} \cot \theta ;
\end{gathered}
$$

there are two solutions for $\theta$ differing by $\pi / 2$, and we choose the solution for which $\sigma_{a}>\sigma_{b}>0$. These equations, which we call Gaussian estimators, are related to the $\mathrm{AB}$ estimator (eq. [5]), which in this notation is simply $\tan \theta=\sigma_{x y} /\left(\sigma_{x x}-\epsilon_{x}^{2}\right)$. However, the Gaussian estimators have the advantages that (1) they are symmetric in $x$ and $y$ and (2) they account naturally for the possibility that there is an intrinsic dispersion $\sigma_{b}$ in the $M_{\mathrm{BH}}-\sigma$ correlation. The Gaussian estimators can easily be extended to include measurement errors that differ from galaxy to galaxy and to provide uncertainties in the estimators (e.g., Gull 1989; see Feigelson \& Babu 1992 for a general review of linear regression procedures), and with these extensions they are likely to provide a more reliable slope estimator than either the $\chi^{2}$ or AB estimators.

We close this section with a general comment on fitting linear relations such as equation (1). The choice of the reference value $\sigma_{0}$ affects the uncertainty in $\alpha$ and the covariance between the estimated values of $\alpha$ and $\beta$. A rough rule of thumb is that $\sigma_{0}$ should be chosen near the middle of the range of values of $\sigma$ in the galaxy sample to minimize the uncertainty in $\alpha$ and the correlation between $\alpha$ and $\beta$. As an example, Ferrarese \& Merritt (2000) use $\sigma_{0}=1 \mathrm{~km} \mathrm{~s}^{-1}$ and find an uncertainty in $\alpha$ of \pm 1.3 . However, most of this uncertainty arises because errors in $\alpha$ and $\beta$ are strongly correlated at this value of $\sigma_{0}$ (correlation coefficient $r=-0.998$ ). Simply by choosing $\sigma_{0}=200 \mathrm{~km} \mathrm{~s}^{-1}$, the uncertainty in $\alpha$ is reduced by a factor of more than 10 , to \pm 0.09 .

\section{THE DATA}

The $M_{\mathrm{BH}}-\sigma$ relation has been explored in the literature using a number of distinct data sets:

1. Sample FM1.--Much of FM's analysis is based on a set of 12 galaxies with "secure" black hole mass estimates (sample A, Table 1 of Ferrarese \& Merritt 2000). However, their definition of "secure" is not itself secure: in $\S 5$, we reject one of the galaxies in this sample (NGC 4374) because of concerns about the reliability of its mass estimate, and the best estimate of the mass of another (IC 1459) has recently increased by a factor of 6 . Half of the black hole mass estimates in this sample come from gas kinematics, as determined by Hubble Space Telescope (HST) emission-line spectra, and the remainder from stellar and maser kinematics. Unless otherwise indicated, when discussing this sample we use the upper and lower limits to the dispersion and black hole mass given by Ferrarese \& Merritt (2000). ${ }^{13}$ The slope estimators then yield

$$
\beta_{\chi}=4.47 \pm 0.44, \quad \beta_{\mathrm{AB}}=4.81 \pm 0.55 .
$$

The minimum $\chi^{2}$ per degree of freedom is 0.69 , which indicates an acceptable fit; thus, there is no evidence for any intrinsic dispersion in this sample.

2. Sample G1.-The sample used by Gebhardt et al. (2000a) contains 26 galaxies. Of these, the majority (18) of the mass estimates are from axisymmetric dynamical models of the stellar distribution function, based on HST and ground-based absorption-line spectra. All of the galaxies in sample FM1 are contained in this sample except for NGC 3115. The stated rms fractional uncertainty in the black hole masses is 0.22 dex, but following Gebhardt et al. (2000a), we adopt $\epsilon_{y}=0.30$, which yields a minimum $\chi^{2}$ per degree of freedom equal to unity. Gebhardt et al. (2000a) take $\epsilon_{x}=0$, corresponding to negligible uncertainties in the dispersions; this approximation is discussed in $\S 4.1$. The slope estimators then yield

$$
\beta_{\chi}=3.74 \pm 0.30, \quad \beta_{\mathrm{AB}}=3.74 \pm 0.23 .
$$

A maximum-likelihood estimate of the intrinsic dispersion in black hole mass at constant velocity dispersion for this sample is $0.22 \pm 0.05$ dex.

3. Sample FM2.-Merritt \& Ferrarese (2001b) supplement sample FM1 with 10 additional galaxies, mostly taken from Kormendy \& Gebhardt (2001), for a total of 22 galaxies. The stated rms fractional uncertainty in the black hole masses is $0.24 \mathrm{dex}$. The slope estimators yield

$$
\beta_{\chi}=4.78 \pm 0.43, \quad \beta_{\mathrm{AB}}=4.65 \pm 0.49 .
$$

The minimum $\chi^{2}$ per degree of freedom is 1.1 , and there is no evidence for any intrinsic dispersion in the black hole mass.

4. Sample G2.-These are the 22 galaxies listed by Kormendy \& Gebhardt (2001) that are also in sample FM2.

\footnotetext{
${ }^{13}$ The error bars in $x$ and $y$ are given by $\left(\log \sigma_{\text {upper }}-\log \sigma_{\text {lower }}\right) / 2$ and $\left(\log M_{\mathrm{BH} \text {, upper }}-\log M_{\mathrm{BH} \text {, lower }}\right) / 2$, respectively.
} 
By comparing samples FM2 and G2, we can isolate the effects of different treatments of the same galaxies. We assume $20 \%$ uncertainty in the dispersion of the Milky Way and $5 \%$ uncertainty in the velocity dispersions of external galaxies (see $\S \S 4.1$ and 4.3). Using G2's stated uncertainties in the black hole masses, the slope estimators yield $\beta_{\chi}=3.70 \pm 0.20, \beta_{\mathrm{AB}}=3.61 \pm 0.31$. The minimum $\chi^{2}$ per degree of freedom is 2.8 , which suggests that either the uncertainties in the black hole masses are underestimated or there is an intrinsic dispersion in black hole mass. Adding an intrinsic dispersion of 0.17 dex decreases the value of $\chi^{2}$ per degree of freedom to unity and reduces the best-fit slope to

$$
\beta_{\chi}=3.61 \pm 0.29, \quad \beta_{\mathrm{AB}}=3.61 \pm 0.31 .
$$

A maximum-likelihood estimate of the intrinsic dispersion in black hole mass at constant velocity dispersion for this sample is $0.16 \pm 0.05$ dex.

\section{WHY ARE THE SLOPES DIFFERENT?}

Our goal is to determine why different investigations yield such a wide range of slopes. In particular, the two samples from FM give slopes $\gtrsim 4.5$ ("high" slopes) with both the $\chi^{2}$ and $\mathrm{AB}$ estimators, while the two samples from the Nukers give slopes $\lesssim 4.0$ ("low" slopes) with both estimators. In $\S \S 4.1-4.4$ we describe several explanations for the slope range that have been proposed in the literature, all of which are found to be inadequate. In $\S 4.5$ we suggest that systematic differences in the dispersions used by FM and the Nukers are responsible for most of the slope discrepancy.

\subsection{Measurement Errors in Velocity Dispersion}

Merritt \& Ferrarese (2001a) argue that random measurement errors in the velocity dispersion can have a significant effect on the slope of the $M_{\mathrm{BH}}-\sigma$ regression. In particular, they claim that the Nukers' assumption of zero measurement error in $\sigma$ leads them to underestimate the slope. To test this claim, we plot in Figure 2 the slope $\beta$ derived from the G1 sample using both the $\mathrm{AB}$ and $\chi^{2}$ estimators, as a function of the assumed rms error $\epsilon_{x}$ in the log of the velocity dispersion.

For nearly all of the galaxies in sample G1, the data typically have signal-to-noise ratios around 100 , and the formal uncertainties in the dispersions are around 2\%-3\% $\left(\epsilon_{x}=0.009-0.013\right)$. However, at this level, stellar template variations, assumptions about the continuum shape, fitting regions used, and atmospheric seeing conditions all can have a noticeable effect on the estimated dispersion. To account crudely for these systematic errors, we double the uncertainties in the dispersions, to $5 \%\left(\epsilon_{x}=0.021\right)$. The uncertainty is larger in the Milky Way (see $\S 4.3$ ) and in a few galaxies that we have not observed ourselves and that do not have accurate dispersion profiles in the literature. The statement of Merritt \& Ferrarese (2001a) that velocitydispersion errors are "easily at the $10 \%$ level" is indeed correct for the sample FM1, where the rms fractional error in the dispersions is $14 \%\left(\epsilon_{x}=0.057\right)$, but their dispersions are mostly based on heterogeneous data that are 20-30 years old (Davies et al. 1987).

Figure 2 shows that the effect of random errors in the dispersions is negligible: at the $5 \%$ level, the change in $\beta$ for

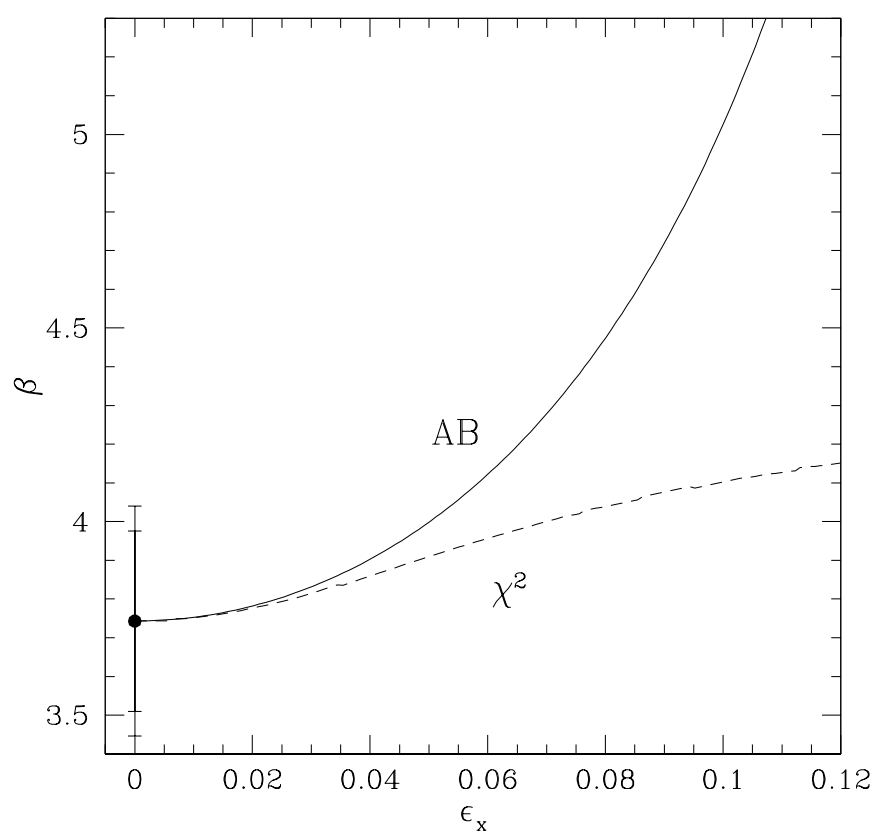

FIG. 2.-Dependence of the slope $\beta$ on the assumed measurement uncertainty in velocity dispersion for the sample G1. The abscissa is the rms measurement error in $\log \sigma$. Solid and dashed lines show the slopes derived from the $\mathrm{AB}$ and $\chi^{2}$ estimators, respectively. The error bars show the computed uncertainty in the slope at zero error. The formal uncertainty in the dispersion measurements of G1 is $\epsilon_{x} \simeq 0.01$; allowing for possible systematic errors in the stellar template and continuum subtraction increases $\epsilon_{x}$ to $\sim 0.02(5 \%)$.

sample G1 is only 0.03 and 0.04 for the $\chi^{2}$ and $A B$ estimators, respectively, and even at the $10 \%$ level the corresponding changes are only 0.12 and 0.16 .

\subsection{Measurement Errors in Black Hole Mass}

We next ask whether the combined effects of varying assumptions about measurement errors in both velocity dispersion and black hole mass can explain the discrepancy between the high and low slopes. As usual, we parameterize these uncertainties by $\epsilon_{x}$ and $\epsilon_{y}$, the rms measurement error in the log of the velocity dispersion and black hole mass. For simplicity, in this subsection these errors are assumed to be the same for all galaxies in each sample. The effects of these uncertainties on the slope $\beta$ can then be explored using the Gaussian estimators (eq. [7]). These estimators have two advantages over the $\chi^{2}$ or $A B$ estimators for this purpose: (1) the slope estimator depends only on the difference $\epsilon_{y}^{2}-\epsilon_{x}^{2}$ and hence is a function of only one variable and (2) the condition that the derived intrinsic dispersion $\sigma_{b}^{2}$ be positive-definite provides an upper limit to the allowable errors.

The left and right panels of Figure 3 show the slope $\beta$ and the maximum allowed value of $\epsilon_{x}$ for each of the galaxy samples in $\S 3$. For each sample there is a minimum slope $\beta$ and a maximum value of $\epsilon_{y}^{2}-\epsilon_{x}^{2}$, beyond which the intrinsic dispersion $\sigma_{b}^{2}$ is negative. In particular, for sample FM1 the minimum allowable slope is $\beta=4.39$; thus, there are no assumptions about the measurement errors that can lead to a slope in the low range. The slope versus error lines in the left panel of Figure 3 are approximately parallel for all four samples; thus, there is no single set of measurement errors that could remove the discrepancy between the high slopes found by FM and the low slopes found by the Nukers. 

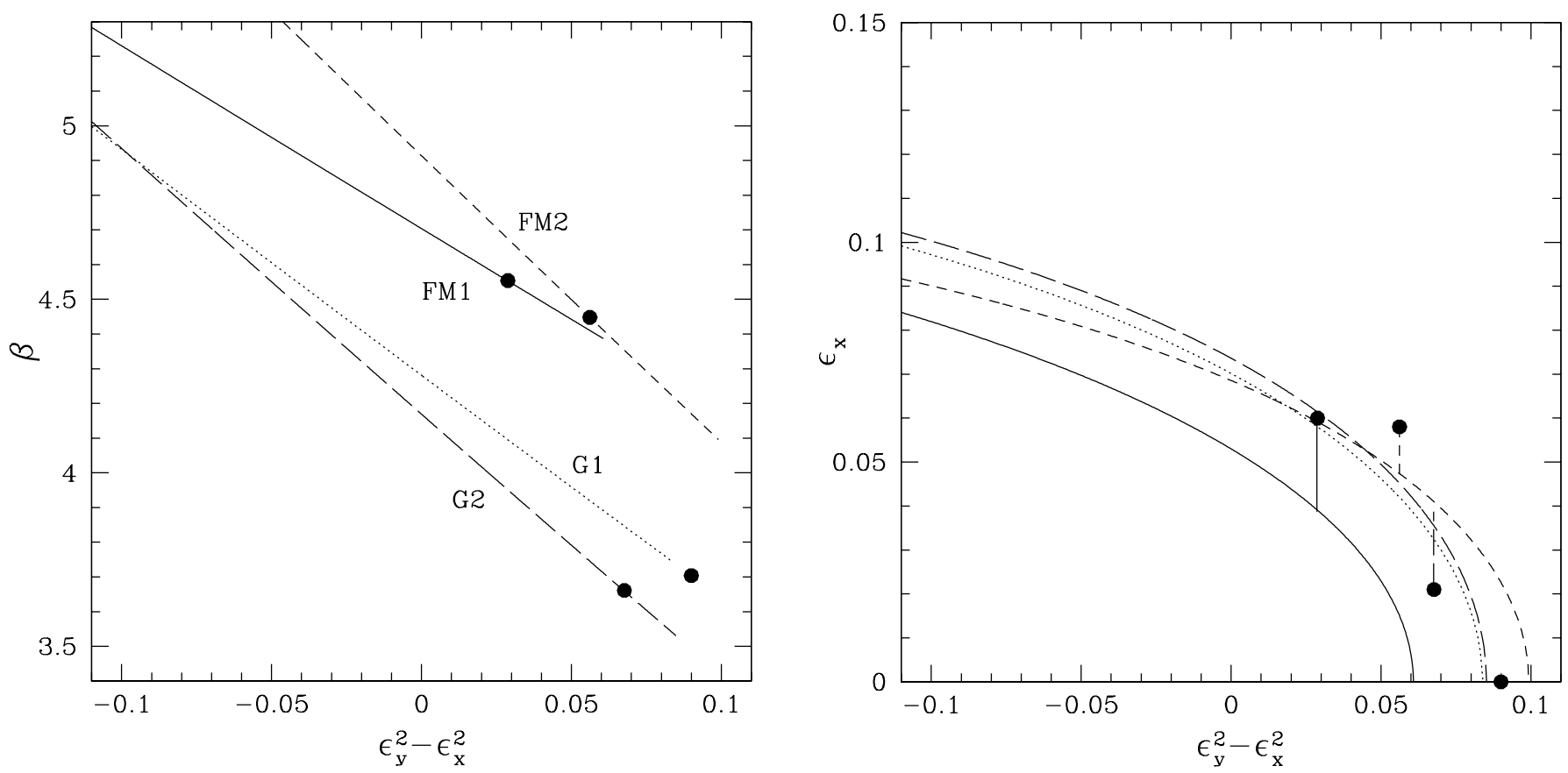

Fig. 3.-Dependence of the slope $\beta$ on the assumed rms errors in black hole mass and velocity dispersion. The rms errors in $\log M_{\mathrm{BH}}$ and $\log \sigma$ are $\epsilon_{y}$ and $\epsilon_{x}$, respectively (assumed the same for all galaxies). The left panel shows the slope derived from the Gaussian estimator (eq. [7]) for samples FM1 (solid line), FM2 (short-dashed line), G1 (dotted line), and G2 (long-dashed line). The lines stop where the intrinsic dispersion $\sigma_{b}^{2}<0$. The right panel shows the maximum allowed value of $\epsilon_{x}$; for larger values the intrinsic dispersion is negative. The filled circles denote the locations corresponding to the estimated values of $\epsilon_{x}$ and $\epsilon_{y}$ in each survey; in the right panel these are connected by vertical lines to the curves for the corresponding survey.

Consistent slopes would require that $\left(\epsilon_{y}^{2}-\epsilon_{x}^{2}\right)_{\text {Nuker }}$ $\simeq\left(\epsilon_{y}^{2}-\epsilon_{x}^{2}\right)_{\mathrm{FM}}-0.07$. This relation, combined with the constraint $\sigma_{b}^{2}>0$, cannot be satisfied with any plausible combination of measurement errors - note in particular that $\epsilon_{y}$ should be similar for the two groups since they rely on many of the same black hole mass determinations, and $\epsilon_{x}$ should be smaller for the Nuker samples than the FM samples, since the Nukers employ high signal-to-noise ratio slit spectra while FM rely on central velocity dispersions from the pre-1990 literature. We conclude that random measurement errors cannot explain the slope discrepancy.

\subsection{The Dispersion of the Milky Way}

Merritt \& Ferrarese (2001a) also argue that the slope is strongly affected by the assumed dispersion for the Milky Way Galaxy, for which the Nukers' estimated dispersion $\sigma=75 \mathrm{~km} \mathrm{~s}^{-1}$ should be increased to $\sigma=100 \mathrm{~km} \mathrm{~s}^{-1}$. We show in Figure 4 how the derived slope depends on the Milky Way dispersion, for both samples G1 and FM1. We see that in fact $\beta$ is quite insensitive to the Milky Way dispersion used in the G1 sample: increasing the dispersion from 75 to $100 \mathrm{~km} \mathrm{~s}^{-1}$ as suggested by Merritt \& Ferrarese (2001a) increases $\beta$ only by 0.13 . The corresponding slope change is substantially larger for sample FM $1-0.27$ for the $\chi^{2}$ estimator and 0.44 for the $\mathrm{AB}$ estimator-but this strong sensitivity reflects the small size of that sample and is not relevant to conclusions drawn by Gebhardt et al. (2000a) from sample G1.

Despite this conclusion, it is worthwhile to determine a more accurate value for the Milky Way dispersion to use in the $M_{\mathrm{BH}}-\sigma$ relation. We review the data on the dispersion of the Galactic bulge in the Appendix, where our results are summarized in the dispersion profile of Figure 9 and equa- tion (A3). We stress that the dispersion profile of the Milky Way is determined from a heterogeneous set of tracers with uneven spatial coverage and by very different methods than the dispersions of the external galaxies discussed in this paper. We therefore assign our estimates of the Milky Way dispersion an uncertainty of $20 \%$, much larger than the formal uncertainty and much larger than the 5\% uncertainty that we assume for the dispersions of external galaxies.

The conversion of the dispersion profile in equation (A3) to a characteristic dispersion is different for FM and the Nukers. FM define their dispersion to be the luminosityweighted rms line-of-sight dispersion within a circular aperture of radius $r_{e} / 8$, where $r_{e}$ is the effective radius. For $r_{e}=0.7 \mathrm{kpc}$ as derived in the Appendix, we find $\sigma=95 \mathrm{~km}$ $\mathrm{s}^{-1}$. Because the bulge is triaxial, we correct the dispersion that we measure from our particular location to the average over all azimuths in the Galactic plane. Binney, Gerhard, \& Spergel (1997) model the bulge as a triaxial system with axis ratios $1: 0.6: 0.4$ and long axis at an angle $\phi_{0}=20^{\circ}$ from the Sun-center line. If the density is stratified on similar ellipsoids, the ratio $r^{2} \equiv \sigma^{2}\left(\phi_{0}=20^{\circ}\right) /\left\langle\sigma^{2}\left(\phi_{0}\right)\right\rangle$ depends only on the axis ratios (Roberts 1962). For the axis ratios given by Binney et al., $r=1.07$. Thus, our best estimate for the dispersion within $r_{e} / 8$ is $\sigma_{\mathrm{FM}}=90 \pm 18 \mathrm{~km} \mathrm{~s}^{-1}$; if we use this instead of FM's estimate of $\sigma=100 \pm 20 \mathrm{~km} \mathrm{~s}^{-1}$, the slope derived from sample FM1 is reduced from $\beta_{\mathrm{AB}}=4.81 \pm 0.55$ to $\beta_{\mathrm{AB}}=4.66 \pm 0.42$ and for sample $\mathrm{FM} 2$ from $\beta_{\mathrm{AB}}=4.65 \pm 0.49$ to $4.54 \pm 0.40$.

In contrast, the Nukers use the luminosity-weighted rms line-of-sight dispersion within a slit aperture of half-length $r_{e}$. This dispersion depends weakly on the slit width, which we take to be $70 \mathrm{pc}$ (corresponding to $1^{\prime \prime}$ at Virgo). In this case we find $\sigma=110 \mathrm{~km} \mathrm{~s}^{-1}$; reducing this by a factor $r$ to account for triaxiality, we have $\sigma=103 \pm 20 \mathrm{~km} \mathrm{~s}^{-1}$, close 

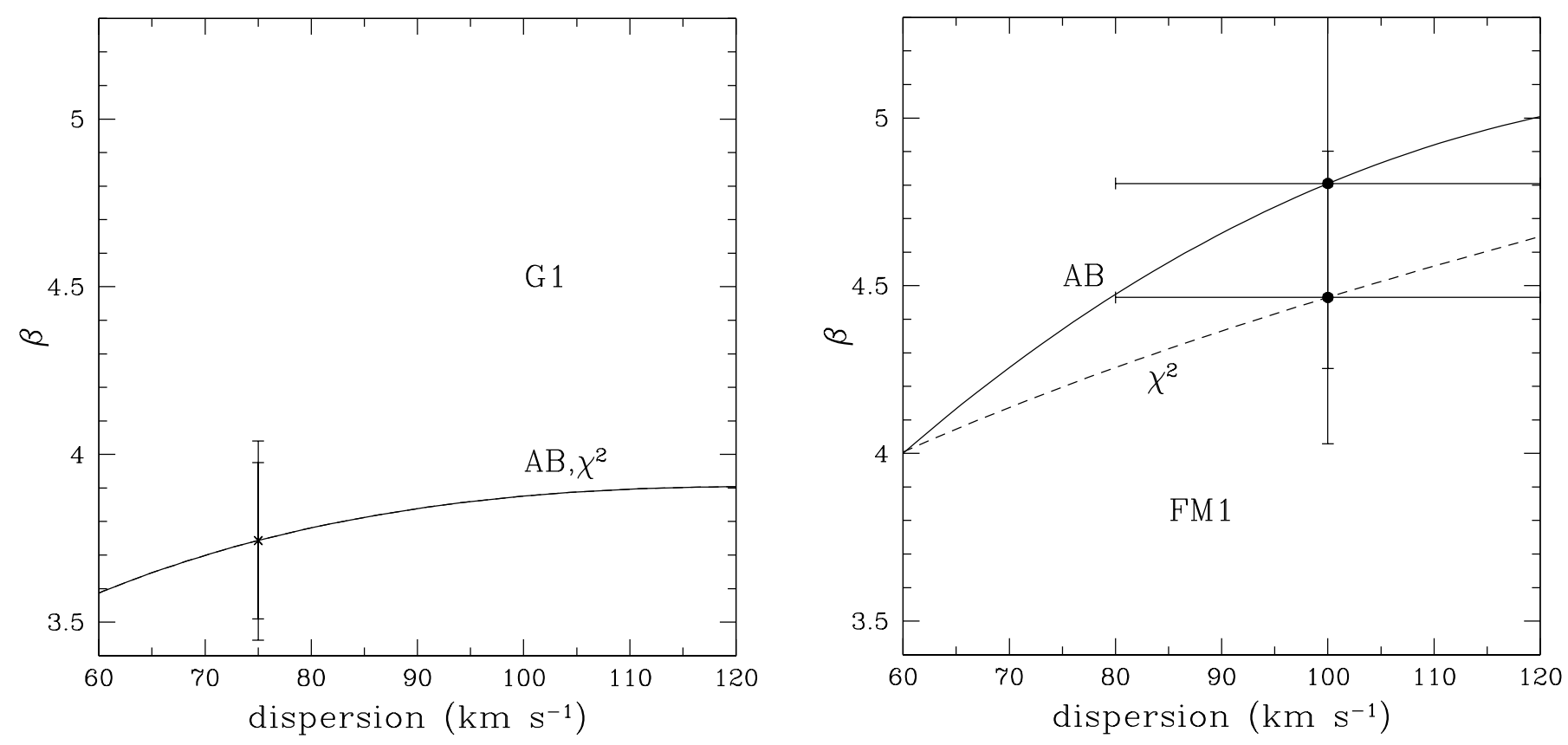

FIG. 4.-Dependence of the slope $\beta$ on the assumed velocity dispersion for the Milky Way in samples G1 (left panel) and FM1 (right panel). The filled circles and error bars show the assumed dispersion and the corresponding slope and error bars. Solid and dashed lines show the slopes derived from the $\mathrm{AB}$ and $\chi^{2}$ estimators, respectively; these are the same for the G1 sample because $\epsilon_{x}=0$ and $\epsilon_{y}$ is the same for all galaxies.

to the value advocated by FM. This change increases the slope derived by Gebhardt et al. (2000a) from $\beta_{\chi}=3.74 \pm 0.15$ only to $\beta_{\chi}=3.88 \pm 0.15$. Thus, improved estimates of the velocity dispersion of the Milky Way bulge reduce the slope discrepancy only slightly.

\subsection{Different Samples}

Merritt \& Ferrarese (2001b) argue that the shallower slope obtained by the Nukers arises in part from the inclusion of galaxies in which the black hole sphere of influence is not well resolved. However, the samples FM2 and G2 contain exactly the same 22 galaxies, all of which are claimed by Merritt \& Ferrarese (2001b) to have a well-resolved sphere of influence, and the difference in slope $\beta_{\chi}$ (eqs. [10] and [11]) is actually larger than that between the samples FM1 and G1.

\subsection{Aperture and Effective Dispersions}

Why, then, are the slopes different, particularly in the samples FM2 and G2, which contain the same galaxies? If we fit the dispersions in these samples to a relation of the form

$$
\log \sigma_{\mathrm{G} 2}=\gamma+(1+\delta) \log \sigma_{\mathrm{FM} 2}
$$

we find

$$
\delta_{\chi}=0.13 \pm 0.10, \quad \delta_{\mathrm{AB}}=0.23 \pm 0.10,
$$

significantly different from the value $\delta=0$ that should obtain if there were no systematic differences between the dispersions (see Fig. 5). A relation of this kind implies that the slopes $\beta$ determined from the FM2 and G2 samples will be related by $\beta_{\mathrm{FM} 2} / \beta_{\mathrm{G} 2}=1+\delta$. Then if $\delta$ is in the range $0.15-0.20$ and the Nuker sample gives $\beta=4$, the sample from FM will give $\beta=4.6-4.8$, well inside the high range. Thus, it appears that the major cause of the range of slopes is systematic differences in the dispersions: FM's dispersions lead to high slopes, and the Nukers' dispersions lead to low slopes.

This possibility was suggested by Gebhardt et al. (2000a) but was later rejected by Merritt \& Ferrarese (2001a), who

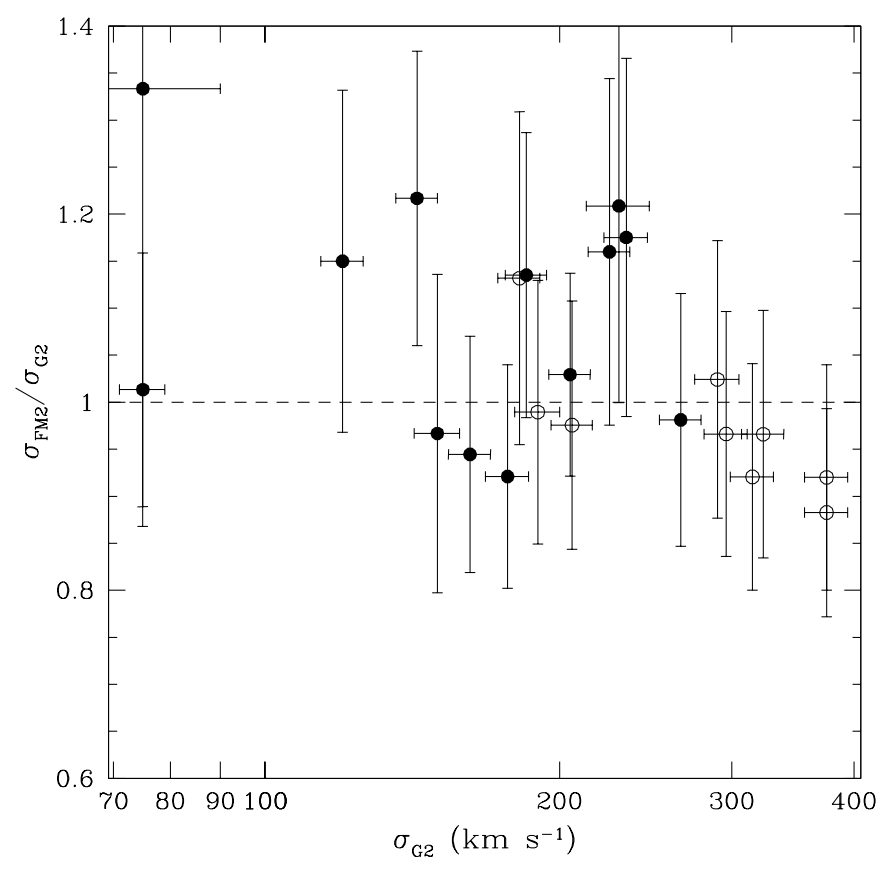

FIG. 5.-Comparison of the velocity dispersions in the samples FM2 and G2. The dispersion ratio $\sigma_{\mathrm{FM} 2} / \sigma_{\mathrm{G} 2}$ is plotted against $\sigma_{\mathrm{G} 2}$. Filled circles denote power-law galaxies, and open circles denote core galaxies. We take the uncertainties in the FM2 dispersions from Merritt \& Ferrarese (2001b) and assume that the uncertainties in the G2 dispersions are $20 \%$ for the Milky Way and 5\% for external galaxies. The uncertainties in dispersion ratio are computed by assuming that the errors in the FM2 dispersions and the G2 dispersions are independent. The plot shows that FM's dispersions are higher than Nuker dispersions at low dispersion and lower at high dispersion. 
argued that systematic differences between dispersions are unimportant because there was "remarkably little difference on average" between the dispersions (they quote a mean ratio of 1.01 and a correlation coefficient in the logs of $0.97)$. However, these statistics have no bearing on the slope $1+\delta$ in equation (12).

There are several possible explanations of the difference in dispersions:

1. The Nukers use the rms dispersion within a slit aperture of length $2 r_{e}$ (hereafter $\sigma_{1}$ ), while FM's results are based on the rms dispersion within a circular aperture of radius $r_{e} / 8$ (hereafter $\sigma_{8}$ ). In practice, these dispersions measure similar spatial scales, since for typical galaxies most of the contribution to the luminosity-weighted dispersion comes from radii near the radius of the circular aperture or the width of the slit aperture, which are typically $1^{\prime \prime}-2^{\prime \prime}$. Nevertheless, the ratio $\sigma_{8} / \sigma_{1}$ could depend systematically on the velocity dispersion of the galaxy, for example,

$$
\sigma_{1} \propto \sigma_{8}^{1+\delta_{1}}
$$

In this case the difference in slope estimates would reflect the structural properties of the galaxies, and the slope of the $M_{\mathrm{BH}}-\sigma$ relation would depend on which dispersion measure was used.

2. FM do not actually measure $\sigma_{8}$. Instead, they use the central velocity dispersion (hereafter $\sigma_{c}$ ), typically measured in an aperture of radius $r_{\mathrm{ap}} \simeq 2^{\prime \prime}$ (Davies et al. 1987), and correct this to a circular aperture of radius $r_{e} / 8$ using the relation (Jørgensen, Franx, \& Kjaergaard 1995) $\sigma_{8}^{\prime}=\sigma_{c}\left(8 r_{\mathrm{ap}} / r_{e}\right)^{0.04}$ (the prime is used to distinguish this approximation to $\sigma_{8}$ from the actual value of $\sigma_{8}$ ). The ratio $\sigma_{8}^{\prime} / \sigma_{8}$ could depend systematically on the velocity dispersion of the galaxy; for example,

$$
\sigma_{8} \propto\left(\sigma_{8}^{\prime}\right)^{1+\delta_{2}} .
$$

Such a trend could arise if the correction factor depends systematically on galaxy luminosity or type, so that the use of the mean relation given by Jørgensen et al. (1995) leads to dispersion-dependent systematic errors. Once again, this would imply that the true slope of the $M_{\mathrm{BH}}-\sigma$ relation would depend on which dispersion measure was used. Alternatively, there could be a universal dispersion profile, but one that is not accurately modeled by the Jørgensen et al. formula; in this case a trend could arise if the ratio $r_{\mathrm{ap}} / r_{e}$ depends systematically on dispersion (the direction of this trend is not obvious: high-dispersion galaxies have larger effective radii but are also further away). In either case the difference in slope estimates would reflect a shortcoming in FM's analysis rather than a real physical effect.

3. The dispersion measurements used by one or both of the two groups could be subject to dispersion-dependent systematic errors (e.g., one set of measurements is systematically low at high dispersions). In this case the difference in slope estimates would reflect problems with the data reduction.

To explore these possibilities, we have examined a sample of 40 early-type galaxies for which Faber et al. (1997) have compiled effective radii and central velocity dispersions and have fitted HST photometry to a five-parameter "Nuker law" profile. Each galaxy is assumed to contain a central black hole, with mass given by the $M_{\mathrm{BH}}-\sigma$ relation in the form derived below (eq. [19]). We use the Nuker law and the assumptions of spherical symmetry, constant mass-to-light ratio, isotropic velocity dispersion, and 1 " slit width to compute the ratios $\sigma_{8} / \sigma_{1}$ and $\sigma_{8}^{\prime} / \sigma_{8}$. This approach is model dependent but has the advantages that (1) the discussion is independent of observational errors in the dispersions, since the dispersion ratios are determined by a dynamical model, and (2) the sample is larger, since more galaxies have $H S T$ photometry than dispersion profiles. We find

$$
\begin{aligned}
\log \left(\frac{\sigma_{8}}{\sigma_{1}}\right)= & (0.004 \pm 0.002) \\
& +(0.021 \pm 0.010) \log \left(\frac{\sigma_{c}}{200 \mathrm{~km} \mathrm{~s}^{-1}}\right), \\
\log \left(\frac{\sigma_{8}^{\prime}}{\sigma_{8}}\right)= & -(0.012 \pm 0.003) \\
& -(0.056 \pm 0.014) \log \left(\frac{\sigma_{c}}{200 \mathrm{~km} \mathrm{~s}^{-1}}\right) .
\end{aligned}
$$

The first of these equations suggests that there is a small but significant systematic trend in the ratio $\sigma_{8} / \sigma_{1}$ with dispersion, of the form (14) with $\delta_{1} \simeq-0.02$. However, this trend has the wrong sign and only a small fraction of the amplitude required to explain the systematic differences in equations (12) and (13); thus, explanation 1 in the list above does not appear to be important.

The second of these equations suggests that there is a larger systematic trend in the ratio $\sigma_{8}^{\prime} / \sigma_{8}$ with dispersion, of the form (15) with $\delta_{2} \simeq 0.06$. This trend is sufficient to explain about one-third of the systematic differences seen in equations (12) and (13) and has a plausible explanation: Gebhardt et al. (1996) and Faber et al. (1997) show that the shape of the surface-brightness profile in the central parts of early-type galaxies depends on the galaxy luminosity (and hence on its velocity dispersion). Thus, the use of a single empirical formula to correct from $\sigma_{c}$ to $\sigma_{8}$ will lead to systematic errors that are correlated with velocity dispersion. It is always better to use the actual kinematic observations, as was done in G1, than to apply empirical correction factors.

Explanation 3, dispersion-dependent systematic measurement errors, is more difficult to assess. Hudson et al. (2001) compare dispersion measurements from 27 sources, including the catalog used by FM (Davies et al. 1987), and in most cases find no evidence for dispersion-dependent errors of the amplitude found in equation (13) (see Hudson et al.'s Fig. 3). Nevertheless, it is striking that the data points in Figure 5 appear sharply lower for dispersions $\gtrsim 300 \mathrm{~km} \mathrm{~s}^{-1}$ than for smaller dispersions. Measuring large dispersions is particularly difficult because the spectral lines blend together. The principal conclusion is that we badly need a systematic campaign of accurate HST and ground-based measurements of the radial velocity-dispersion profiles of early-type galaxies with black hole candidates. A second conclusion is that the slope of the $M_{\mathrm{BH}}-\sigma$ relation should be estimated only from dispersion measurements at or within well-defined metric radii rather than from central velocity dispersions measured within apertures of a given angular radius.

The same sample of 40 galaxies can also be used to explore the degree of contamination of the dispersions by the dynamical influence of the central black hole. We computed spherical, isotropic dynamical models with and without a black hole of mass given by equation (19). We denote the ratio of the dispersion $\sigma_{1}$ with and without the black hole by $f_{1}$ and the analogous ratio for the dispersion $\sigma_{8}^{\prime}$ by 


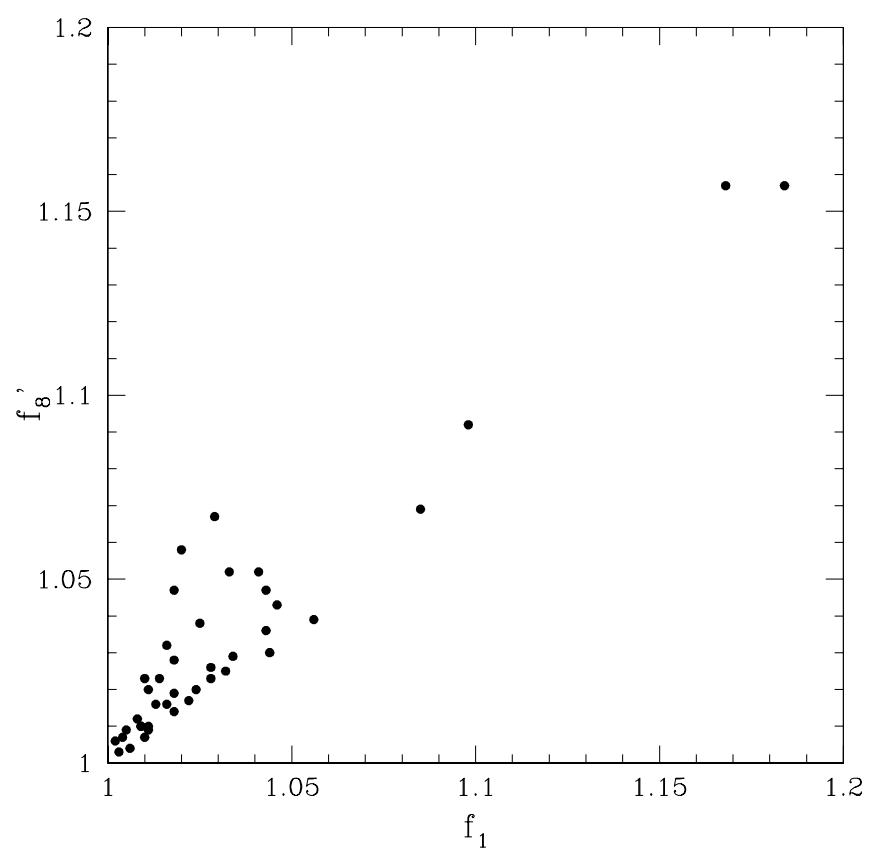

Fig. 6.-Effect of central black holes on measured dispersions. For 40 early-type galaxies listed in Faber et al. (1997), we have computed isotropic, spherical dynamical models that match the surface-brightness distributions and mass-to-light ratios given in that paper. For each galaxy two models are computed: one with no central black hole and one with a black hole of mass given by eq. (19). The abscissa represents the ratio of the dispersions $\sigma_{1}$ in these two models, where $\sigma_{1}$ is the luminosity-weighted rms line-ofsight dispersion within a slit aperture of half-length $r_{e}$ and width $1^{\prime \prime}$ (used by the Nukers). The ordinate represents the ratio of the dispersions $\sigma_{8}^{\prime}$; here $\sigma_{8}^{\prime}$ is an approximation to the dispersion within a circular aperture of radius $r_{e} / 8$, obtained from the central dispersion using an empirical correction formula (used by FM). The galaxies in the upper right corner are M32 and NGC 3115.

$f_{8}^{\prime}$. The results are shown in Figure 6. In general, the ratios $f_{1}$ and $f_{8}^{\prime}$ are similar, reflecting the fact that the two dispersion measures are averaged over similar spatial scales (the slit width for $f_{1}$ and the aperture radius for $f_{8}^{\prime}$ ). In most galaxies the addition of the black hole raises either dispersion no more than 3\%-4\%. However, in a few cases the contamination is much larger, more than $15 \%$. In such galaxies the dispersion measures $\sigma_{c}, \sigma_{1}, \sigma_{8}$, and $\sigma_{8}^{\prime}$ are all misleading. Future versions of the $M_{\mathrm{BH}}-\sigma$ relation should be based on dispersion measures that are less strongly weighted to the center.

\section{BLACK HOLE MASS VERSUS VELOCITY DISPERSION: A NEW ESTIMATE}

In this section we present a new analysis of the $M_{\mathrm{BH}}-\sigma$ relation using the 31 galaxies in Table 1; over half of these have new or revised black hole mass or dispersion determinations since the analysis by Gebhardt et al. (2000a).

\subsection{Comments on Individual Galaxies}

Milky Way.-We use the black hole mass estimate by Chakrabarty \& Saha $(2001),\left(1.8_{-0.3}^{+0.4}\right) \times 10^{6} M_{\odot}$. For comparison, Ghez et al. (1998) find $(2.4 \pm 0.2) \times 10^{6} M_{\odot}$, and Genzel et al. (2000) find (2.6-3.3) $\times 10^{6} M_{\odot}$. The dispersion and its uncertainty are discussed in $\S 4.3$. The bulge mass-tolight ratio is taken from Kent (1992).

M32.- The velocity dispersion is obtained from van der Marel et al. (1994), and the black hole mass,
$M_{\mathrm{BH}}=(2.5 \pm 0.5) \times 10^{6} M_{\odot}$, is from Verolme et al. (2002). In estimating the dispersion, we have excluded the region near the center that is strongly perturbed by the black hole (see Fig. 6). Other recent mass estimates, by van der Marel et al. (1998) and Joseph et al. (2001), give similar results: $(3.9 \pm 0.8) \times 10^{6}$ and $(3 \pm 1) \times 10^{6} M_{\odot}$, respectively.

M31.- The modeling is complicated by the double nucleus. Kormendy \& Bender (1999) find $M_{\mathrm{BH}}=(3.0 \pm 1.5) \times 10^{7} M_{\odot}$, although this result relies heavily on the small displacement between the center of light of the nucleus and bulge. Tremaine (1995) and Bacon et al. (2001) find $M \simeq 7 \times 10^{7} M_{\odot}$ but without detailed model fitting. We adopt the range $(2.0-8.5) \times 10^{7} M_{\odot}$.

$N G C$ 1023.- Both the velocity dispersion and black hole mass are from Bower et al. (2001).

$N G C$ 1068.-The black hole mass is taken from Greenhill \& Gwinn (1997); the error estimates are our own and are very approximate. The dispersion (Kobulnicky \& Gebhardt 2000 ) is somewhat uncertain because of contamination from the bright nucleus.

$N G C 3115$. - The black hole mass is based on stellar kinematics (Kormendy et al. 1996a). Although NGC 3115 does not have three-integral axisymmetric dynamical models, it does have a compact, high-contrast stellar nucleus, and the mass of the nucleus plus black hole can be estimated from the virial theorem. In estimating the dispersion, we have excluded the region near the center that is strongly perturbed by the black hole (see Fig. 6). See also Emsellem, Dejonghe, \& Bacon (1999)

NGC 3245.-The velocity dispersion is obtained from Simien \& Prugniel (1998).

NGC 4258.- The velocity dispersion is obtained from Héraudeau \& Simien (1998).

$N G C 4342$. - Both the velocity dispersion and the black hole mass are from Cretton \& van den Bosch (1999).

$N G C 4486$. - The mass is the average of the values given by Harms et al. (1994) and Macchetto et al. (1997), corrected to a distance of $16.1 \mathrm{Mpc}$.

$N G C$ 2787, NGC 4459, NGC 4596.-The black hole masses are based on Space Telescope Imaging Spectrograph (STIS) measurements of ionized-gas disks by Sarzi et al. (2001). The disk inclinations are determined from dust-lane morphology. Note that the distance and dispersion for NGC 2787, 7.5 Mpc and $140 \mathrm{~km} \mathrm{~s}^{-1}$, are much smaller than the values assumed by Sarzi et al. (2001). Our distance is from Tonry et al. (2001), and the dispersion was measured by one of us (Gebhardt). For NGC 4459 and NGC 4596, we have used the dispersions $\sigma_{8}^{\prime}$ from Sarzi et al. (2001), since on average these are close to $\sigma_{1}$ (eq. [16]).

$N G C$ 7052.- The velocity dispersion is from van den Bosch \& van der Marel (1995).

IC 1459. - The mass estimate that we use (Cappellari et al. 2002), based on stellar kinematics, is much larger than an earlier estimate by the same group from gas kinematics, $(2-6) \times 10^{8} M_{\odot}$ (Verdoes Kleijn et al. 2000). The mass estimate from stellar kinematics is much more reliable, since the gas rotation curve is asymmetric and non-Keplerian. The dispersion also comes from Cappellari et al. (2002).

We do not include the following galaxies in our sample:

NGC 4594 (Kormendy et al. 1996b), NGC 4486B (Kormendy et al. 1997), NGC 4350 (Pignatelli, Salucci, \& Danese 2001), NGC $3031=$ M81, and NGC 3998 (Bower et 
TABLE 1

GALAXY SAMPLE

\begin{tabular}{|c|c|c|c|c|c|c|c|c|}
\hline Galaxy & Type & $M_{B}$ & $\begin{array}{c}M_{\mathrm{BH}}(\text { Low, High }) \\
\left(M_{\odot}\right)\end{array}$ & Method & $\begin{array}{c}\sigma_{1} \\
\left(\mathrm{~km} \mathrm{~s}^{-1}\right)\end{array}$ & $\begin{array}{l}\text { Distance } \\
(\mathrm{Mpc})\end{array}$ & $M / L$, Band & $\begin{array}{c}\text { References for } \\
\text { Black Hole Masses }\end{array}$ \\
\hline Milky Way ............ & $\mathrm{SBbc}$ & -17.65 & $1.8 \times 10^{6}(1.5,2.2)$ & $\mathrm{s}, \mathrm{p}$ & 103 & 0.008 & $1.0, K$ & 1 \\
\hline $\mathrm{N} 221=\mathrm{M} 32 \ldots \ldots \ldots$ & E2 & -15.83 & $2.5 \times 10^{6}(2.0,3.0)$ & s, 3I & 75 & 0.81 & $1.85, I$ & 2 \\
\hline $\mathrm{N} 224=\mathrm{M} 31 \ldots \ldots$. & $\mathrm{Sb}$ & -19.00 & $4.5 \times 10^{7}(2.0,8.5)$ & $\mathrm{s}$ & 160 & 0.76 & $5, V$ & $3,4,5$ \\
\hline 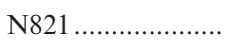 & E4 & -20.41 & $3.7 \times 10^{7}(2.9,6.1)$ & s, 3I & 209 & 24.1 & $5.8, V$ & 6,7 \\
\hline N1023 ................. & SB0 & -18.40 & $4.4 \times 10^{7}(3.9,4.9)$ & s, 3I & 205 & 11.4 & $5.0, V$ & 8 \\
\hline 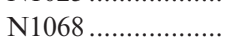 & $\mathrm{Sb}$ & -18.82 & $1.5 \times 10^{7}(1.0,3.0)$ & $\mathrm{m}$ & 151 & 15.0 & & 9 \\
\hline 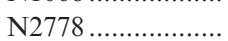 & E2 & -18.59 & $1.4 \times 10^{7}(0.5,2.2)$ & s, 3I & 175 & 22.9 & $6.4, V$ & 6,7 \\
\hline 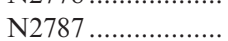 & SB0 & -17.28 & $4.1 \times 10^{7}(3.6,4.5)$ & $\mathrm{g}$ & 140 & 7.5 & $\ldots$ & 10 \\
\hline $\mathrm{N} 3115 \ldots \ldots \ldots \ldots \ldots \ldots$ & So & -20.21 & $1.0 \times 10^{9}(0.4,2.0)$ & $\mathrm{s}$ & 230 & 9.7 & $6.9, \mathrm{~V}$ & 11 \\
\hline N3245 ................. & S0 & -19.65 & $2.1 \times 10^{8}(1.6,2.6)$ & g & 205 & 20.9 & $3.7, R$ & 12 \\
\hline N3377 …........................... & E5 & -19.05 & $1.0 \times 10^{8}(0.9,1.9)$ & s, $3 \mathrm{I}$ & 145 & 11.2 & $2.7, \mathrm{~V}$ & 6,13 \\
\hline 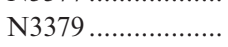 & E1 & -19.94 & $1.0 \times 10^{8}(0.5,1.6)$ & s, 3I & 206 & 10.6 & $4.6, V$ & 14 \\
\hline N3384 …................... & So & -18.99 & $1.6 \times 10^{7}(1.4,1.7)$ & s, 3I & 143 & 11.6 & $2.8, \mathrm{~V}$ & 6,7 \\
\hline 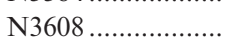 & E2 & -19.86 & $1.9 \times 10^{8}(1.3,2.9)$ & s, 3I & 182 & 22.9 & $3.7, V$ & 6,7 \\
\hline 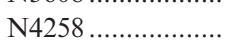 & $\mathrm{Sbc}$ & -17.19 & $3.9 \times 10^{7}(3.8,4.0)$ & $\mathrm{m}, \mathrm{a}$ & 130 & 7.2 & $\ldots$ & 15 \\
\hline 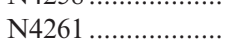 & E2 & -21.09 & $5.2 \times 10^{8}(4.1,6.2)$ & g & 315 & 31.6 & $5.0, V$ & 16 \\
\hline N4291 & E2 & -19.63 & $3.1 \times 10^{8}(0.8,3.9)$ & s, 3I & 242 & 26.2 & $4.4, V$ & 6,7 \\
\hline 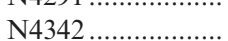 & S0 & -17.04 & $3.0 \times 10^{8}(2.0,4.7)$ & s, 3I & 225 & 15.3 & $6.3, I$ & 17 \\
\hline 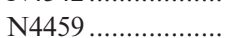 & S0 & -19.15 & $7.0 \times 10^{7}(5.7,8.3)$ & $\mathrm{g}$ & 186 & 16.1 & 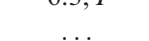 & 10 \\
\hline 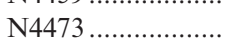 & E5 & -19.89 & $1.1 \times 10^{8}(0.31,1.5)$ & $\mathrm{s}, 3 \mathrm{I}$ & 190 & 15.7 & $6.3, V$ & 6,7 \\
\hline $\mathrm{N} 4486=\mathrm{M} 87 \ldots \ldots$ & E0 & -21.53 & $3.0 \times 10^{9}(2.0,4.0)$ & $\mathrm{g}$ & 375 & 16.1 & $4.0, V$ & 18,19 \\
\hline N4564 ................. & E3 & -18.92 & $5.6 \times 10^{7}(4.8,5.9)$ & $\mathrm{s}, 3 \mathrm{I}$ & 162 & 15.0 & $1.9, I$ & 6,7 \\
\hline N4596 .............................. & SB0 & -19.48 & $7.8 \times 10^{7}(4.5,12)$ & g & 152 & 16.8 & & 10 \\
\hline 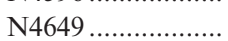 & E1 & -21.30 & $2.0 \times 10^{9}(1.4,2.4)$ & $\mathrm{s}, 3 \mathrm{I}$ & 385 & 16.8 & $9.0, \mathrm{~V}$ & 6,7 \\
\hline N4697 ….................................... & E4 & -20.24 & $1.7 \times 10^{8}(1.6,1.9)$ & $\mathrm{s}, 3 \mathrm{I}$ & 177 & 11.7 & $4.8, V$ & 6,7 \\
\hline 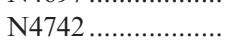 & E4 & -18.94 & $1.4 \times 10^{7}(0.9,1.8)$ & $\mathrm{s}, 3 \mathrm{I}$ & 90 & 15.5 & $\ldots$ & 20 \\
\hline N5845 .................. & E3 & -18.72 & $2.4 \times 10^{8}(1.0,2.8)$ & $\mathrm{s}, 3 \mathrm{I}$ & 234 & 25.9 & $4.8, V$ & 6 \\
\hline N6251 ................. & E2 & -21.81 & $5.3 \times 10^{8}(3.5,7.0)$ & g & 290 & 93.0 & $8.5, V$ & 21 \\
\hline 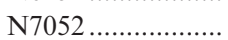 & E4 & -21.31 & $3.3 \times 10^{8}(2.0,5.6)$ & g & 266 & 58.7 & $6.3, I$ & 22 \\
\hline N7457 …............................ & S0 & -17.69 & $3.5 \times 10^{6}(2.1,4.6)$ & $\mathrm{s}, 3 \mathrm{I}$ & 67 & 13.2 & $3.4, V$ & 6,7 \\
\hline IC $1459 \ldots \ldots \ldots \ldots \ldots$ & E3 & -21.39 & $2.5 \times 10^{9}(2.1,3.0)$ & s, 3I & 340 & 29.2 & $3.1, I$ & 23 \\
\hline
\end{tabular}

NoTES.-Distances are taken from Tonry et al. 2001 for most of the galaxies; where these are not available, the distance is determined from the recession velocity, assuming a Hubble constant of $80 \mathrm{~km} \mathrm{~s}^{-1} \mathrm{Mpc}^{-1}$. Absolute magnitudes are for the hot component of the galaxy only. The mass-to-light ratios $M / L$ are usually determined from the same dynamical models that are used to derive the black hole masses; they are given here for reference but play no role in our analysis. Methods: $\mathrm{s}=$ stellar radial velocities; $\mathrm{p}=$ stellar proper motions; $\mathrm{m}=$ maser radial velocities; $\mathrm{a}=$ maser accelerations; $\mathrm{g}=$ rotating gas disk from emission-line observations; $3 \mathrm{I}=$ axisymmetric dynamical models, including three integrals of motion.

RefEREnCES.- (1) Chakrabarty \& Saha 2001; (2) Verolme et al. 2002; (3) Tremaine 1995; (4) Kormendy \& Bender 1999; (5) Bacon et al. 2001; (6) Gebhardt et al. 2002; (7) J. Pinkney et al. 2002, in preparation; (8) Bower et al. 2001; (9) Greenhill \& Gwinn 1997; (10) Sarzi et al. 2001; (11) Kormendy et al. 1996a; (12) Barth et al. 2001b; (13) Kormendy et al. 1998; (14) Gebhardt et al. 2000b; (15) Herrnstein et al. 1999; (16) Ferrarese, Ford, \& Jaffe 1996; (17) Cretton \& van den Bosch 1999; (18) Harms et al. 1994; (19) Macchetto et al. 1997; (20) M. E. Kaiser et al. 2002, in preparation; (21) Ferrarese \& Ford 1999; (22) van der Marel \& van den Bosch 1998; (23) Cappellari et al. 2002.

al. 2000) exhibit strong evidence from stellar dynamics for a black hole but do not yet have three-integral dynamical models.

NGC 4374 (M84) has strong evidence for a black hole from gasdynamics, but the published estimates of the black hole mass differ by far more than the stated errors: Bower et al. (1998) find (0.9-2.6) $\times 10^{9} M_{\odot}$; Maciejewski \& Binney (2001) find $4 \times 10^{8} M_{\odot}$, and a preliminary estimate from Barth et al. (2001a) is $10^{9} M_{\odot}$. The mass assigned to this galaxy is a factor of 4 larger in sample FM2 than in sample G2, which is by far the largest discrepancy between the two samples.

NGC 4945 has a mass estimate from maser emission (Greenhill, Moran, \& Herrnstein 1997) but no reliable dispersion.

NGC 5128 has a mass estimate from ground-based observations of a rotating gas disk (Marconi et al. 2001) but no $H S T$ spectroscopy; moreover, the galaxy has peculiar morphology, presumably because of a recent merger, and thus may not follow the same $M_{\mathrm{BH}}-\sigma$ relation as more normal galaxies

Our sample contains eight galaxies with black hole mass estimates based on gas kinematics. We have some concern that these results may have large systematic errors, due in part to uncertainties in the spatial distribution of the gas (e.g., filled disk or torus configuration, uncertain inclination and thickness) and the large but uncertain correction for pressure support. We therefore urge caution when interpreting results from samples in which a large fraction of the black hole mass estimates are based on gas kinematics. Eventually, galaxies with black hole mass determinations from more than one technique will be invaluable for disentangling the systematic errors in different methods.

\subsection{Slope Estimation}

We use the sample of galaxies and black hole masses in Table 1 to estimate the logarithmic slope $\beta$ in the $M_{\mathrm{BH}}-\sigma$ relation. We assume $20 \%$ uncertainty in the dispersion of 
the Milky Way (see $\S 4.3$ ) and 5\% uncertainties in the dispersions of external galaxies (see $\S 4.1$ ), although the uncertainties in the dispersions of a few galaxies that we have not observed ourselves may be larger. Initially, we assume 0.33 dex rms uncertainties in the black hole masses, which yields $\chi^{2}$ per degree of freedom of unity. Using the $\chi^{2}$ and $A B$ estimators defined in $\S 2$, we find

$$
\beta_{\chi}=4.03 \pm 0.33, \quad \beta_{\mathrm{AB}}=4.12 \pm 0.34 .
$$

This approach does not account for the varying precision of the mass estimates for different galaxies. Therefore, we have also computed the slope using the estimated errors in the black hole masses in Table 1, adding to these in quadrature a common intrinsic dispersion with rms value $\epsilon_{0}$ [i.e., $\left.\epsilon_{y i} \rightarrow\left(\epsilon_{y i}^{2}+\epsilon_{0}^{2}\right)^{1 / 2}\right]$. We find that $\epsilon_{0}=0.27$ gives

$$
\beta_{\chi}=4.00 \pm 0.31, \quad \beta_{\mathrm{AB}}=4.12 \pm 0.34,
$$

with minimum $\chi^{2}$ per degree of freedom of 1.00. A maximum-likelihood estimate of the intrinsic dispersion in black hole mass at constant velocity dispersion for this sample is $\epsilon_{0}=0.23 \pm 0.05$ dex.

In both equations (17) and (18) the $\mathrm{AB}$ estimator for the slope is larger than the $\chi^{2}$ estimator by about 0.1 ; since we have shown in $\S 2$ that the $\mathrm{AB}$ estimator may be biased, we prefer to rely on the $\chi^{2}$ estimator. For our final answer we simply average $\beta_{\chi}$ from equations (17) and (18). Including results for the parameter $\alpha$ obtained in the same way, we have

$$
\alpha=8.13 \pm 0.06, \quad \beta=4.02 \pm 0.32 ;
$$

the parameter $\alpha$ is evaluated for $\sigma_{0}=200 \mathrm{~km} \mathrm{~s}^{-1}$, for which the correlation coefficient between $\alpha$ and $\beta$ is only -0.09 .

Thus, our best estimate (19) is just at the edge of the low range, $\beta \lesssim 4.0$. To test the robustness of this result, we have tried culling the sample in several ways:

1. If we consider only the 21 galaxies from Table 1 with masses determined from stellar kinematics, we find

$$
\alpha=8.13 \pm 0.09, \quad \beta=4.02 \pm 0.44 ;
$$

the close agreement in the parameters in equations (19) and (20) implies that there is no significant systematic bias between masses determined by stellar kinematics and other methods.

2. The dispersions for the Milky Way and for external galaxies are determined by quite different methods. The Milky Way also has one of the smallest and most accurate black hole masses in our sample, and therefore has an unusually strong influence on the slope of the $M_{\mathrm{BH}}-\sigma$ relation. If we remove the Milky Way from our sample, the slope is reduced to $\beta=3.88 \pm 0.32$, a change of 0.14 ( 0.4 standard deviations) toward even lower slopes.

2. We have argued in $\S 4.5$ that high velocity-dispersion measurements may be subject to systematic errors. Thus, we also estimate the slope using only the 25 galaxies in the sample with dispersion less than $250 \mathrm{~km} \mathrm{~s}^{-1}$. We find $\beta=3.77 \pm 0.49$; once again the slope is even lower than our best estimate (19).

3 . The galaxy sample with the most homogeneous observations and analysis consists of the 10 galaxies analyzed by J. Pinkney et al. (2002, in preparation) and Gebhardt et al. (2002). These all have HST spectra acquired with STIS as well as ground-based spectra, HST photometry, and axi- symmetric orbit-based dynamical models and were all reduced and analyzed in the same way. For this sample we find $\beta=3.67 \pm 0.70$; once again the slope is consistent with and even lower than our best estimate.

4. We have removed nine galaxies from the sample that were subject to criticism: the Milky Way (uncertain dispersion), M31 (no accurate models of the double nucleus), NGC 1068 (both the dispersion and the interpretation of the maser kinematics are uncertain), NGC 2778 (the lowest signal-to-noise ratio in the Gebhardt et al. 2002 sample and a correspondingly large uncertainty in the black hole mass), NGC 3115 (no three-integral dynamical models), NGC 3379 and NGC 5845 (these have only a single Faint Object Spectrograph pointing rather than STIS slit spectra at $H S T$ resolution; while there is no obvious problem with either measurement, other galaxies in the Gebhardt et al. 2002 sample have superior spatial coverage of the kinematics), NGC 4459 (the inclination of the gas disk is uncertain because the kinematic data come from a single long-slit spectrum; also, the dispersion is uncertain because it is obtained from low-resolution data), and NGC 6251 (the most distant galaxy with a black hole mass measurement; the sphere of influence of the black hole is poorly resolved; the mean velocities do not clearly show Keplerian rotation; and, in addition, there are the usual uncertainties - uncertain disk orientation, influence of random motions in the gas-associated with mass measurements from gas kinematics). The reduced sample of 22 galaxies has a slope $\beta=3.79 \pm 0.32$, once again lower than our best estimate.

Since most of these culled samples have slopes that are smaller than our best fit (19), we suspect that our best fit may slightly overestimate the true slope by $0.1-0.3$.

The data from Table 1 and the fit (19) are shown in Figure 7. In Figure 8 we show the residuals to the best-fit correlation.

The two largest residuals in Figure 7 belong to NGC 2778 $(-0.75$ dex $)$ and the Milky Way $(-0.72$ dex $)$; the largest positive residual belongs to NGC 3115 (+0.63 dex). The poor fit of the Milky Way probably arises because its dispersion profile has been determined by methods different from those for the other galaxies, using heterogeneous tracers and a variety of surveys; we have allowed for this by assigning the Milky Way dispersion an uncertainty of $20 \%$, compared to $5 \%$ for external galaxies. The large residual in NGC 3115 may arise because its mass has been estimated by simply applying the virial theorem to its nucleus rather than by dynamical modeling. The large residual in NGC 2778 may reflect the low signal-to-noise ratio of its kinematic data (J. Pinkney et al. 2002, in preparation).

\section{CONCLUSIONS}

The masses $M_{\mathrm{BH}}$ of dark objects ("black holes") in the centers of nearby early-type galaxies are related to the velocity dispersion $\sigma$ by the loglinear relation (1). We have used the sample of 31 galaxies in Table 1 to determine the parameters in this relation, where $\sigma$ is defined to be the luminosityweighted $\mathrm{rms}$ velocity dispersion in a slit extending to the effective radius. Our best estimate for the slope of this relationship is $4.0 \pm 0.3$ (eq. [19]), although several culled, and perhaps higher quality, samples give slopes that are lower by $0.1-0.3$. There is no evidence for systematic differences in either slope or normalization between black hole mass 


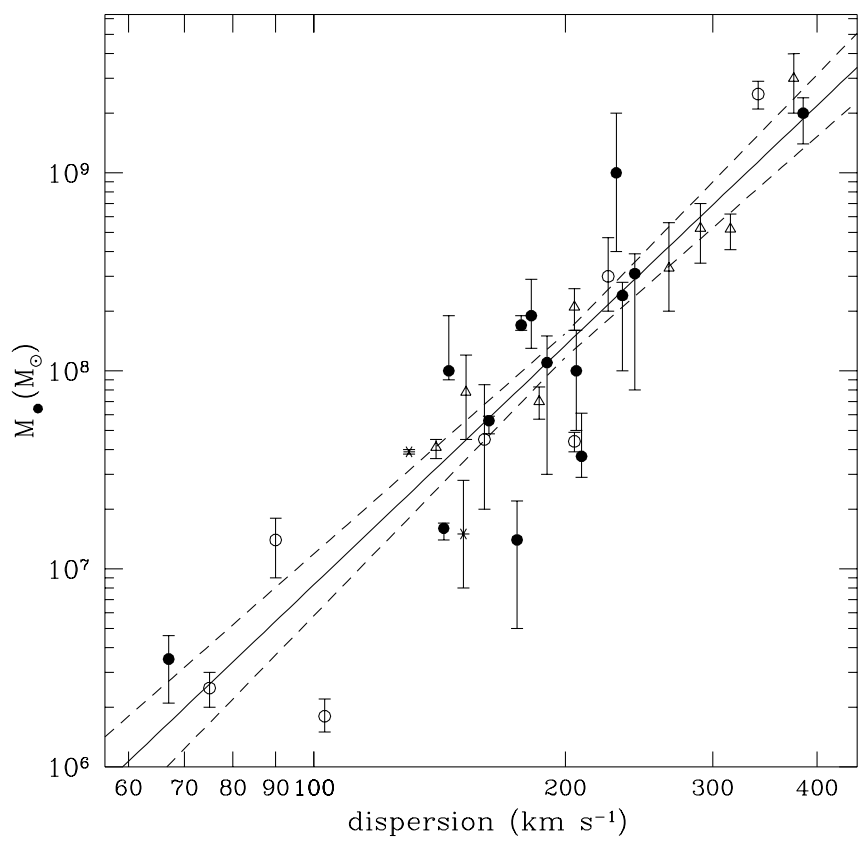

Fig. 7.-Data on black hole masses and dispersions for the galaxies in Table 1, along with the best-fit correlation described by eqs. (1) and (19). Mass measurements based on stellar kinematics are denoted by circles, on gas kinematics by triangles, and on maser kinematics by asterisks; Nuker measurements are denoted by filled circles. The dashed lines show the $1 \sigma$ limits on the best-fit correlation.

measurements based on stellar kinematics and gas kinematics. If the stated measurement errors in the black hole masses are correct or if they are underestimated because of systematic errors, the intrinsic dispersion in the $M_{\mathrm{BH}^{-}} \sigma$ relation is no larger than about $0.25-0.3$ dex in black hole mass (i.e., less than a factor of 2 ).

Black hole mass estimates based on gas kinematics are particularly uncertain, due to uncertainties in the spatial distribution of the gas (e.g., filled disk or torus configuration, uncertain inclination and thickness) and the large but uncertain correction for pressure support. In particular, including a correction for pressure support will increase the black hole mass; since four of the six high-dispersion galaxies in our sample have masses determined by gas kinematics, a systematic increase in their masses could increase the best-fit slope.

The range of slopes for the $M_{\mathrm{BH}}-\sigma$ relation found in the literature appears to arise mostly from systematic differences in the velocity dispersions used by different groups. We do not believe that these differences reflect the different definitions of dispersion used by the groups (FM use the dispersion within a circular aperture of radius $r_{e} / 8$, and the Nukers use the dispersion within a slit aperture of halflength $r_{e}$ ). It appears that part of the difference results from Ferrarese \& Merritt's analysis, in which central velocity dispersions are extrapolated to $r_{e} / 8$ using an empirical formula. However, another - and possibly larger-component appears to arise from poorly understood systematic errors in the dispersion measurements.

In a few galaxies, the influence of the central black hole may significantly affect the velocity dispersions-both the central dispersions used by FM and the slit dispersions used

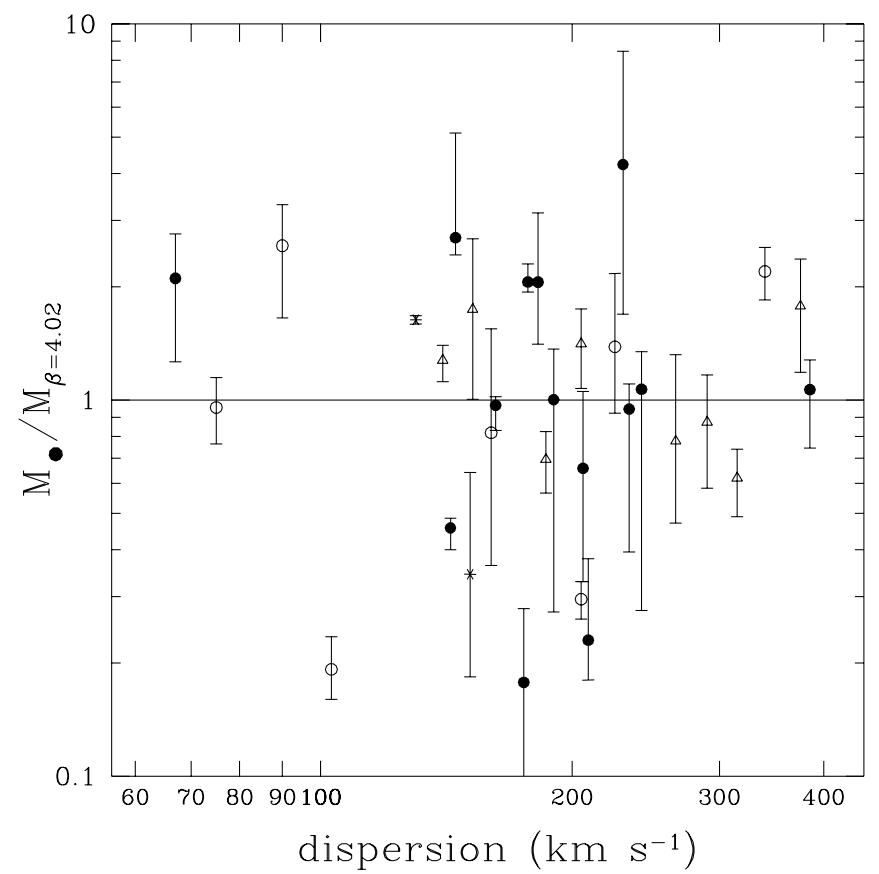

FIG. 8. - Residuals between the black hole masses and dispersions for the galaxies in Table 1 and the best-fit correlation described by eq. (1) with $\beta=4.02$ (eq. [19]). Mass measurements based on stellar kinematics are denoted by circles, on gas kinematics by triangles, and on maser kinematics by asterisks; Nuker measurements are denoted by filled circles.

by the Nukers. Future analyses of the $M_{\mathrm{BH}}-\sigma$ relation should be based on velocity-dispersion measures that are less strongly weighted to the center; it is likely that both the slope and the intrinsic scatter of the relation depend on which dispersion measure is used, and it will be interesting to seek the dispersion measure that offers the smallest intrinsic scatter. Other improvements in the analysis would include the use of statistical estimators that are more robust and that explicitly include an intrinsic dispersion in the black hole mass, accounting properly for the asymmetric error bars in black hole mass determinations, and estimating more accurately the uncertainties in individual dispersion measurements.

The investment of the astronomy community in the difficult task of measuring black hole masses has not yet been matched by a commensurate investment in the much easier task of obtaining high-quality kinematic maps of galaxies containing black holes. A complete set of high-quality dispersion and rotation profiles for the galaxies in Table 1 would allow us to explore more deeply how the black hole mass is related to the kinematic structure of its host galaxy.

We thank Michael Hudson and Tim de Zeeuw for discussions and Tim de Zeeuw for communicating results in advance of publication. Support for proposals 7388, 8591, 9106, and 9107 was provided by NASA through a grant from the Space Telescope Science Institute, which is operated by the Association of Universities for Research in Astronomy, Inc., under NASA contract NAS 5-26555. This research was also supported by NSF grant AST 99-00316. 


\section{APPENDIX}

\section{THE EFFECTIVE DISPERSION FOR THE MILKY WAY}

The Milky Way has one of the most accurate black hole masses and anchors the low-mass end of the $M_{\mathrm{BH}^{-}} \sigma$ relation. Therefore, it is important to have an accurate value for the dispersion of the Milky Way bulge.

The first task is to estimate the effective or half-light radius $r_{e}$ of the bulge. Kent (1992) models Spacelab K-band observations of the bulge with a major-axis emissivity profile of the form

$$
j(a)= \begin{cases}j_{i} a^{-1.85} & a<0.94 \mathrm{kpc}, \\ j_{0} K_{0}\left(a / a_{0}\right) & a>0.94 \mathrm{kpc}\end{cases}
$$

where $K_{0}$ is a modified Bessel function, $a_{0}=0.67 \mathrm{kpc}$, and the constants $j_{i}$ and $j_{0}$ are chosen so that the emissivity is continuous. In a spherical galaxy described by equation (A1), the effective radius is $1.50 a_{0}$ or $1.0 \mathrm{kpc}$; Kent's model is oblate and axisymmetric, with axis ratio 0.6 , so the geometric mean of the three effective semiaxes is smaller by $(0.6)^{1 / 3}$, yielding $r_{e}=0.84 \mathrm{kpc}$.

Dwek et al. (1995) fit COBE measurements in several bands to a wide variety of triaxial models for the emissivity. Their best-fit model at $K$ band has a Gaussian emissivity profile with an effective semimajor axis of $1.86 \mathrm{kpc}$; the axis ratios are $1: 0.4: 0.3$, so our best estimate for the effective radius is $r_{e}=1.86 \mathrm{kpc}(0.4 \times 0.3)^{1 / 3}=0.92 \mathrm{kpc}$. Their second-best model (E3) has $j(a) \propto K_{0}\left(a / a_{0}\right)$ and an effective radius $r_{e}=0.56 \mathrm{kpc}$.

Binney et al. (1997) use COBE L-band photometry to perform a disk/bulge decomposition. Their equation (1b) describes an analytic model for the bulge emissivity that fits the data "very well":

$$
j(a)=j_{0} \frac{e^{-a^{2} / a_{m}^{2}}}{\left(1+a / a_{0}\right)^{1.8}},
$$

where $a$ is the semimajor axis and $a_{m}=1.9 \mathrm{kpc}$. They quote $a_{0}=100 \mathrm{pc}$, but this value reflects the fact that the data have been smoothed to an angular resolution of 1.5 or $200 \mathrm{pc}$, and photometry at higher resolution suggests that $a_{0}$ is less than 1 pc (e.g., Genzel et al. 1996). The effective semimajor axis for equation (A2) is $0.48 a_{m}$ or $0.91 \mathrm{kpc}$; the corresponding geometric mean of the effective semiaxes $(1: 0.6: 0.4)$ is $r_{e}=0.57 \mathrm{kpc}$.

Based on these estimates, we adopt $r_{e}=0.7 \pm 0.2 \mathrm{kpc}$. The much larger estimate $r_{e}=2.7 \mathrm{kpc}$ given by Merritt \& Ferrarese (2001a) is based on a table in Gilmore, King, \& van der Kruit (1990), which in turn appears to be based on the galaxy model of Bahcall \& Soneira (1980), which in turn is based on comments by G. de Vaucouleurs in the 1970s that $r_{e}$ is about one-third of the distance of the Sun from the Galactic center.

The next task is to estimate the velocity dispersion as a function of radius. We are interested in the rms line-of-sight velocity $\left\langle v_{\mathrm{LOS}}^{2}\right\rangle^{1 / 2}$ measured relative to the local standard of rest, since this is the closest analog to the dispersions used in the $M_{\mathrm{BH}}-\sigma$ relation for external galaxies. This quantity differs from the usual dispersion quoted in bulge studies, which is relative to the local mean velocity, $\sigma=\left\langle\left(v_{\text {LOS }}-\bar{v}\right)^{2}\right\rangle^{1 / 2}$, where $\bar{v}=\left\langle v_{\text {LOS }}\right\rangle$. When papers quote values for $\sigma$ and $\bar{v}$, we set $\left\langle v_{\text {LOS }}^{2}\right\rangle=\bar{v}^{2}+\sigma^{2}$. We use the following sources:

1. Due to the interest in the black hole in our Galaxy, the kinematics in the central few parsecs have been investigated much more thoroughly than the kinematics at larger radii (Genzel et al. 2000, especially their Fig. 16). The entries at radii less than 5 pc in Table 2 are taken from Genzel et al.'s Table 4; at these radii corrections for rotation are negligible.

2. $\mathrm{OH} / \mathrm{IR}$ stars are mass-losing asymptotic giant branch stars, which are detected by hydroxyl maser emission from their circumstellar envelopes. They are old enough to represent a phase-mixed population and are unaffected by obscuration, and hence should be good tracers of the kinematics of the bulge. The survey by Lindqvist, Habing, \& Winnberg (1992a) and Lindqvist et al. (1992b) lists $133 \mathrm{OH} / \mathrm{IR}$ stars within $1^{\circ}$ or $140 \mathrm{pc}$ of the Galactic center. We have divided these into three equal groups by projected distance from the center and computed the dispersion for each group. One limitation of this survey is that its radial-velocity coverage was relatively small, $\left|v_{\mathrm{LOS}}\right| \leq 217 \mathrm{~km} \mathrm{~s}^{-1}$, so that high-velocity OH/IR stars might have been missed. We have corrected for this cutoff, assuming that the distribution of line-of-sight velocities is Gaussian, in the two bins where the correction is less than $10 \%$ and have discarded the third bin. At larger distances, Sevenster et al. (1997) have located $307 \mathrm{OH} /$ IR stars in the region $|l|<10^{\circ},|b|<3^{\circ}$. The minimum velocity range in this survey was $-330 \mathrm{~km} \mathrm{~s}^{-1}<v_{\mathrm{LOS}}<402$ $\mathrm{km} \mathrm{s}^{-1}$, so velocity selection effects are negligible. We have discarded all sources not having a standard double-peaked profile and all sources with expansion velocity greater than $17 \mathrm{~km} \mathrm{~s}^{-1}$, which appear to represent a younger, more rapidly rotating population (Winnberg, Lindqvist, \& Habing 1998). The remaining 208 stars were divided into five equal groups by projected distance, and the mean projected distance and dispersion were computed for each group.

3. Beaulieu, Dopita, \& Freeman (1999) have conducted an $\mathrm{H} \alpha$ survey for new planetary nebulae and remeasured the velocities of many known planetary nebulae. Their databases contain 183 planetary nebulae within $10^{\circ}$ of the Galactic center. We have divided these into four equal groups by projected distance, and the mean projected distance and dispersion were computed for each group. Beaulieu et al. estimate that their velocity errors are $\pm 11 \mathrm{~km} \mathrm{~s}^{-1}$, which is negligible.

4. Blum et al. $(1994,1995)$ have measured the dispersion of samples of M giants in four fields between 160 and $300 \mathrm{pc}$ from the Galactic center. Terndrup, Sadler, \& Rich (1995) have measured the dispersion of K giants in Baade's window (0.56 kpc from the Galactic center). We include only stars with $V>16.0$, which they believe restricts the sample to bulge stars and eliminates the foreground disk. 
TABLE 2

Velocity Dispersion Measurements in the INNER BULGE $(r<1 \mathrm{kpc})$

\begin{tabular}{|c|c|c|}
\hline $\begin{array}{l}\text { Radius } \\
\text { (pc) }\end{array}$ & $\left\langle v_{\mathrm{LOS}}^{2}\right\rangle^{1 / 2}$ & Reference \\
\hline $0.085 \ldots \ldots \ldots$. & $195 \pm 34$ & 1 \\
\hline 0.33 & $164 \pm 74$ & 1 \\
\hline $0.34 \ldots \ldots \ldots \ldots \ldots \ldots$ & $102 \pm 8$ & 1 \\
\hline $0.39 \ldots \ldots \ldots \ldots \ldots \ldots$ & $99 \pm 10$ & 1 \\
\hline $0.67 .$. & $72 \pm 5$ & 1 \\
\hline 0.78. & $85 \pm 15$ & 1 \\
\hline $1.2 .$. & $68 \pm 13$ & 1 \\
\hline $3.9 .$. & $54 \pm 6$ & 1 \\
\hline 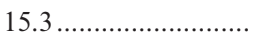 & $70 \pm 7$ & 2 \\
\hline $38.5 \ldots \ldots \ldots \ldots \ldots \ldots \ldots \ldots$ & $101 \pm 11$ & 2 \\
\hline 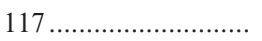 & $126 \pm 14$ & 3 \\
\hline $160 \ldots \ldots \ldots \ldots \ldots \ldots \ldots \ldots$ & $156 \pm 18$ & 4 \\
\hline 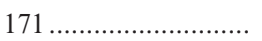 & $128 \pm 14$ & 4 \\
\hline 288 & $129 \pm 14$ & 4 \\
\hline 299 & $148 \pm 19$ & 4 \\
\hline 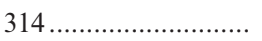 & $130 \pm 14$ & 3 \\
\hline 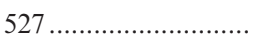 & $101 \pm 11$ & 3 \\
\hline $562 \ldots \ldots \ldots \ldots \ldots \ldots \ldots \ldots$ & $110 \pm 10$ & 5 \\
\hline $612 \ldots \ldots \ldots \ldots \ldots \ldots \ldots \ldots$ & $117 \pm 12$ & 6 \\
\hline 789 & $88 \pm 9$ & 6 \\
\hline 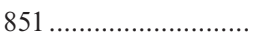 & $102 \pm 12$ & 3 \\
\hline 989 & $100 \pm 10$ & 6 \\
\hline 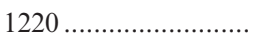 & $89 \pm 9$ & 6 \\
\hline $1284 \ldots \ldots \ldots \ldots \ldots \ldots \ldots \ldots$ & $79 \pm 8$ & 3 \\
\hline
\end{tabular}

REFERENCES.-(1) Genzel et al. 2000; (2) Lindqvist et al. 1992b; (3) Sevenster et al. 1997; (4) Blum et al. 1995; (5) Terndrup et al. 1995; (6) Beaulieu et al. 1999.

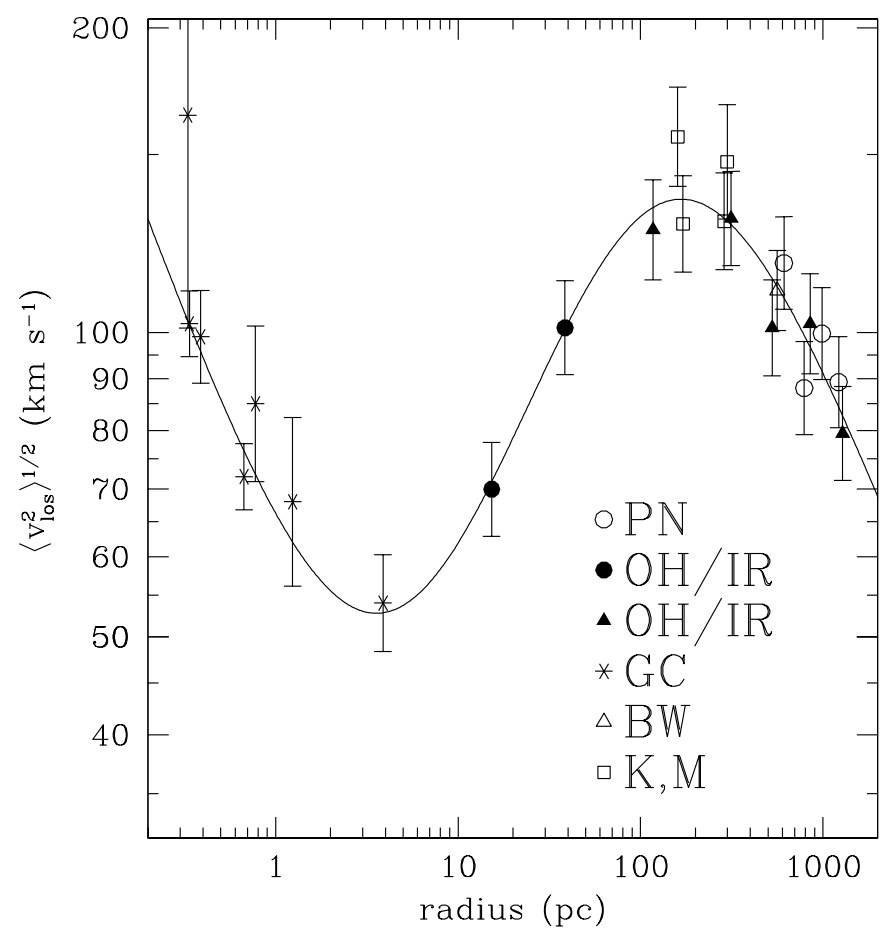

Fig. 9.- The rms line-of-sight velocity in the bulge of the Milky Way, as a function of radius. PN = planetary nebulae (Beaulieu et al. 1999); $\mathrm{OH} / \mathrm{IR}=\mathrm{OH} / \mathrm{IR}$ stars (Lindqvist et al. 1992a, 1992b; Sevenster et al. 1997); BW = giant stars in Baade's window (Terndrup et al. 1995); K, M = giant stars (Blum et al. 1994, 1995); and GC = stars near the Galactic center (Genzel et al. 2000). Filled symbols denote observations biased toward the Galactic plane, and open symbols denote observations biased away from the plane. The curve is the fitting function (A3). 
The data at $r>0.1 \mathrm{pc}$ shown in Figure 9 and Table 2 have been fitted to the functional form

$$
\left\langle v_{\mathrm{LOS}}^{2}\right\rangle^{1 / 2}=c \frac{\left(r / r_{0}\right)^{\alpha}}{1+b\left(r / r_{0}\right)^{\beta}}+d\left(r / r_{0}\right)^{-1 / 2} .
$$

For $r_{0}=500 \mathrm{pc}$ the best-fit values are $c=633 \mathrm{~km} \mathrm{~s}^{-1}, \alpha=0.67, \beta=1.14, b=4.64$, and $d=2.52$. The general features of this curve - a minimum in the dispersion near $5 \mathrm{pc}$ and a maximum of $\sim 130 \mathrm{~km} \mathrm{~s}^{-1}$ at a few hundred parsecs — are not new (Kent 1992).

A possible concern is that the bulge is flattened, with an axis ratio of about 0.5 , so the dispersion at a given radius may depend on the angle between the radius vector and the Galactic plane. To address this concern, we have divided the data points from outside $4 \mathrm{pc}$ from the Galactic center into those biased toward the minor axis, plotted with filled symbols (the criterion is $\langle|l|\rangle>\langle|b|\rangle$, where $l$ and $b$ are the Galactic longitude and latitude; these are objects such as planetary nebulae and late-type giants that are found optically), and those biased toward the major axis (the $\mathrm{OH} / \mathrm{IR}$ stars, found in surveys along the Galactic plane), which are plotted with open symbols. There is no obvious systematic difference between the dispersion curves defined by the filled and open symbols.

We employ the fit (A3) at the end of $\S 4.3$ to estimate the appropriate Milky Way dispersion to use in the $M_{\mathrm{BH}}-\sigma$ relation.

Adams, F. C., Graff, D. S., \& Richstone, D. O. 2001, ApJ, 551, L31 Akritas, M. G., \& Bershady, M. A. 1996, ApJ, 470, 706

Bacon, R., Emsellem, E., Combes, F., Copin, Y., Monnet, G., \& Martin, P. 2001, A\&A, 371, 409

Bahcall, J. N., \& Soneira, R. M. 1980, ApJS, 44, 73

Barth, A. J., Sarzi, M., Ho, L. C., Rix, H.-W., Shields, J. C., Filippenko, A. V., Rudnick, L., \& Sargent, W. L. W. 2001a, in ASP Conf. Ser. 249, The Central Kiloparsec of Starbursts and AGNs, ed. J. H. Knapen, J. K. Beckman, I. Shlosman, \& T. J. Mahoney (San Francisco: ASP), 370

Barth, A. J., Sarzi, M., Rix, H.-W., Ho, L. C., Filippenko, A. V., \& Sargent, W. L. W. 2001b, ApJ, 555, 685

Beaulieu, S., Dopita, M. A., \& Freeman, K. C. 1999, ApJ, 515, 610

Binney, J., Gerhard, O., \& Spergel, D. 1997, MNRAS, 288, 365

Blum, R. D., Carr, J. S., Depoy, D. L., Sellgren, K., \& Terndrup, D. M. 1994, ApJ, 422, 111

Blum, R. D., Carr, J. S., Sellgren, K., \& Terndrup, D. M. 1995, ApJ, 449, 623

Bower, G. A., Wilson, A. S., Heckman, T. M., Magorrian, J., Gebhardt, K., Richstone, D. O., Peterson, B. M., \& Green, R. F. 2000, AAS Meeting, 197, 92.03

Bower, G. A., et al. 1998, ApJ, 492, L111

2001, ApJ, 550, 75

Burkert, A., \& Silk, J. 2001, ApJ, 554, L151

Cappellari, M., Verolme, E. K., van der Marel, R. P., Verdoes Kleijn, G. A., Illingworth, G. D., Franx, M., Carollo, C. M., \& de Zeeuw, P. T. 2002, ApJ, submitted (astro-ph/0202155)

Chakrabarty, D., \& Saha, P. 2001, AJ, 122, 232

Cretton, N., \& van den Bosch, F. 1999, ApJ, 514, 704

Davies, R. L., Burstein, D., Dressler, A., Faber, S. M., Lynden-Bell, D.,

Terlevich, R. J., \& Wegner, G. 1987, ApJS, 64, 581

Dwek, E., et al. 1995, ApJ, 445, 716

Emsellem, E., Dejonghe, H., \& Bacon, R. 1999, MNRAS, 303, 495

Faber, S. M., et al. 1997, AJ, 114, 1771

Fabian, A., \& Iwasawa, K. 1999, MNRAS, 303, L34

Feigelson, E. D., \& Babu, G. J. 1992, ApJ, 397, 55

Ferrarese, L. 2002, in Current High-Energy Emission around Black Holes, ed. C.-H. Lee (Singapore: World Scientific), in press

Ferrarese, L., \& Ford, H. C. 1999, ApJ, 515, 583

Ferrarese, L., Ford, H. C., \& Jaffe, W. 1996, ApJ, 470, 444

Ferrarese, L., \& Merritt, D. 2000, ApJ, 539, L9

Gebhardt, K., et al. 1996, AJ, 112, 105

2000a, ApJ, 539, L13

2000 b, AJ, 119, 1157

2002, ApJ, submitted

Genzel, R., Pichon, C., Eckart, A., Gerhard, O. E., \& Ott, T. 2000,

MNRAS, 317,348

Genzel, R., Thatte, N., Krabbe, A., Kroker, H., \& Tacconi-Garman, L. E. 1996, ApJ, 472, 153

Ghez, A. M., Klein, B. L., Morris, M., \& Becklin, E. E. 1998, ApJ, 509, 678

Gilmore, G., King, I. R., \& van der Kruit, P. C. 1990, The Milky Way as a

Galaxy (Mill Valley: University Science Books)

Greenhill, L. J., \& Gwinn, C. R. 1997, Ap\&SS, 248, 261

Greenhill, L. J., Moran, J. M., \& Herrnstein, J. R. 1997, ApJ, 481, L23

Gull, S. F. 1989, in Maximum Entropy and Bayesian Methods, ed. J. Skilling (Dordrecht: Kluwer), 511

Haehnelt, M. G., \& Kauffmann, G. 2000, MNRAS, 318, L35

Harms, R. J., et al. 1994, ApJ, 435, L35

Héraudeau, P., \& Simien, F. 1998, A\&AS, 133, 317
Herrnstein, J. R., et al. 1999, Nature, 400, 539

Hudson, M. J., Lucey, J. R., Smith, R. J., Schlegel, D. J., \& Davies, R. L. 2001, MNRAS, 327, 265

Jørgensen, I., Franx, M., \& Kjaergaard, P. 1995, MNRAS, 276, 1341

Joseph, C. L., et al. 2001, ApJ, 550, 668

Kendall, M., Stuart, A., \& Ord, J. K. 1983, The Advanced Theory of Statistics, Vol. 3 (4th ed.; London: Charles Griffin)

Kent, S. M. 1992, ApJ, 387, 181

Kobulnicky, H. A., \& Gebhardt, K. 2000, AJ, 119, 1608

Kormendy, J., \& Bender, R. 1999, ApJ, 522, 772

Kormendy, J., Bender, R., Evans, A. S., \& Richstone, D. 1998, AJ, 115, 1823

Kormendy, J., \& Gebhardt, K. 2001, in AIP Conf. Proc. 586, The 20th Texas Symposium on Relativistic Astrophysics, ed. J. C. Wheeler \& H. Martel (Melville: AIP), 363

Kormendy, J., et al. 1996a, ApJ, 459, L57 - 1996b, ApJ, 473, L91 1997, ApJ, 482, L139

Lindqvist, M., Habing, H. J., \& Winnberg, A. 1992a, A\&A, 259, 118

Lindqvist, M., Winnberg, A., Habing, H. J., \& Matthews, H. E. 1992b, A\&AS, 92, 43

Macchetto, F., Marconi, A., Axon, D. J., Capetti, A., Sparks, W., \& Crane, P. 1997, ApJ, 489, 579

Maciejewski, W., \& Binney, J. 2001, MNRAS, 323, 831

Marconi, A., Capetti, A., Axon, D. J., Koekemoer, A., Macchetto, D., \& Schreier, E. J. 2001, ApJ, 549, 915

Merritt, D., \& Ferrarese, L. 2001a, ApJ, 547, 140

2001b, in ASP Conf. Ser. 249, The Central Kiloparsec of Starbursts and AGNs, ed. J. H. Knapen, J. K. Beckman, I. Shlosman, \& T. J. Mahoney (San Francisco: ASP), 335

Ostriker, J. P. 2000, Phys. Rev. Lett., 84, 5258

Pignatelli, E., Salucci, P., \& Danese, L. 2001, MNRAS, 320, 124

Press, W. H., Teukolsky, S. A., Vetterling, W. T., \& Flannery, B. P. 1992, Numerical Recipes (2d ed.; Cambridge: Cambridge Univ. Press)

Roberts, P. H. 1962, ApJ, 136, 1108

Sarzi, M., Rix, H.-W., Shields, J. C., Rudnick, G., Ho, L. C., McIntosh, D. H., Filippenko, A. V., \& Sargent, W. L. W. 2001, ApJ, 550, 65

Sevenster, M. N., Chapman, J. M., Habing, H. J., Killeen, N. E. B., \& Lindqvist, M. 1997, A\&AS, 122, 79

Simien, F., \& Prugniel, P. 1998, A\&AS, 131, 287

Sołtan, A. 1982, MNRAS, 200, 115

Terndrup, D. M., Sadler, E. M., \& Rich, R. M. 1995, AJ, 110, 1774

Tonry, J. L., Dressler, A., Blakeslee, J. P., Ajhar, E. A., Fletcher, A. B., Luppino, G. A., Metzger, M. R., \& Moore, C. B. 2001, ApJ, 546, 681

Tremaine, S. 1995, AJ, 110,628

van den Bosch, F. C., \& van der Marel, R. P. 1995, MNRAS, 274, 884

van der Marel, R. P., Cretton, N., de Zeeuw, P. T., \& Rix, H.-W. 1998, ApJ, 493, 613

van der Marel, R. P., Rix, H.-W., Carter, D., Franx, M., White, S. D. M., \& de Zeeuw, T. 1994, MNRAS, 268, 521

van der Marel, R. P., \& van den Bosch, F. C. 1998, AJ, 116, 2220

Verdoes Kleijn, G. A., van der Marel, R. P., Carollo, M., \& de Zeeuw, P. T. 2000, AJ, 120, 1221

Verolme, E. K., et al. 2002, MNRAS, submitted (astro-ph/0201086)

Winnberg, A., Lindqvist, M., \& Habing, H. J. 1998, in ASP Conf. Ser. 186, The Central Parsecs of the Galaxy, ed. H. Falcke et al. (San Francisco: ASP), 389

$\mathrm{Yu}, \mathrm{Q}$., \& Tremaine, S. 2002, MNRAS, in press 